\title{
UCRL 9999
} MASTER

University of California

\section{Ernest O. Lawrence Radiation Laboratory}

\section{BIBLIOGRAPHY ON PION-PION INTERACTION}

Berkeley, California 


\section{DISCLAIMER}

This report was prepared as an account of work sponsored by an agency of the United States Government. Neither the United States Government nor any agency Thereof, nor any of their employees, makes any warranty, express or implied, or assumes any legal liability or responsibility for the accuracy, completeness, or usefulness of any information, apparatus, product, or process disclosed, or represents that its use would not infringe privately owned rights. Reference herein to any specific commercial product, process, or service by trade name, trademark, manufacturer, or otherwise does not necessarily constitute or imply its endorsement, recommendation, or favoring by the United States Government or any agency thereof. The views and opinions of authors expressed herein do not necessarily state or reflect those of the United States Government or any agency thereof. 


\section{DISCLAIMER}

Portions of this document may be illegible in electronic image products. Images are produced from the best available original document. 
UCRL -9999

UC-34 Phys. Dist.

TID -4500 (17th Ed.)

\section{UNIVERSITY OF CALIFORNIA \\ Lawrence Radiation Laboratory \\ Berkeley, California}

Contract No. W-7405-eng-48

BIBLIOGRAPHY ON PION-PION INTERACTION

M. Lynn Stevenson

November. 7, 1961 

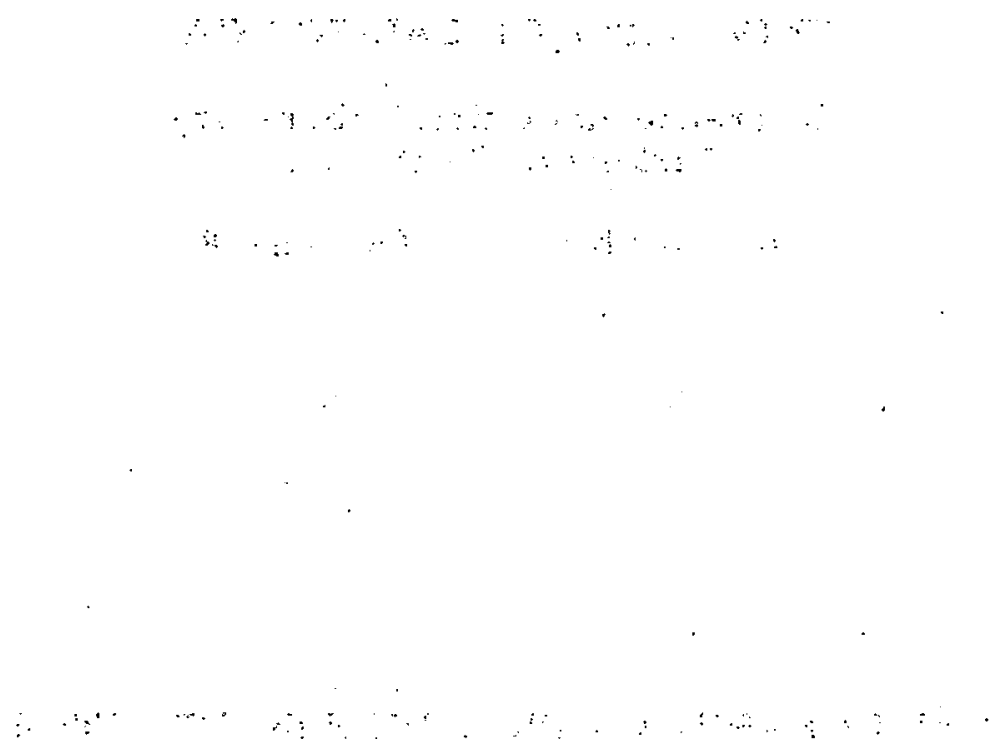

Printed in USA. Price $\$ 1.50$. Available from the office of Technical Services U. S. Department of Commerce Washington 25 , D.C. 


\title{
BIBLIOGRAPHY ON PION-PION INTERACTION
}

\author{
M. Lynn Stevenson \\ Lawrence Radiation Laboratory \\ University of California \\ Berkeley, California
}

November 7, 1961

\section{Foreword}

This bibliography is arranged in three parts.

Part I is a chronological listing of references together with exceipts or comments.

In Part II the references are grouped according to subject.

In Part III the authors' names are listed alphabetically and keyed to the entries in Part I.

The bibliography includes papers on the subject of pion-pion. interactions that were known to.me on November 7, 1961. If there are omissions, I shall appreciate information on them. 
THIS PAGE

WAS INTENTIONALLY

LEFT BLANK 
BIBLIOGRAPHY ON PION-PION INTERACTION

\section{Contents}

Foreword

Part I: CHRONOLOGICAL LISTING

a. Experimental Papers . . . . . . . . . . . . . 1

b. Theoretical Papers . . . . . . . . . . . . . 8

Part II: LISTING BY TOPICS

A. General Theoretical Papers . . . . . . . . . . . 4 4

B. Pion-Nucleon Interaction

a. Experimental Papers : : . . . . . . . . : . 42

b. Theoretical Papers . . . . . . . . . . . . . . 44

C. Nucleon-Antinucleon Interaction

a. Experimental Papers . . . . . . . . . . . 46

b. Theoretical Papers.. . . . . . . . . . . 46

D. Electromagnetic Structure of the Nucleons

a. Experimental Papers . . . . . . . . . . . 47

b. Theoretical Papers . . . . . . . . . . . . . . . . . . . . . . 477

E. $\mathrm{K}^{ \pm}-\mathrm{Mes}$ on Decay

a. Experimental Paper . . . . . . . . . . . . . 48

b. Theoretical Papers . . . . . . . . . . . . . . 48

F. Photoproduction

a. Experimental Papers : : : : . . . . . . . 49

b. Theoretical Papers . . . . . . . . . . . . . 49

G. Heavy Mesons

a. Experimental Paper . . : . : : . . . ... . 50

b. Theoretical Papers . . . . . . . . . . . . . . 50

H. Nucleon-Nucleon Interaction

I. Proton-Deuteron Interaction

a. Experimental Papers . . . . . . . . . . . . 51

b. Theoretical Papers... . . . . . . . . . 51

J. Kaon-Nucleon Interaction

Theoretical Paper . . . . . . : . . . . 52

K. Lack of Evidence

a. Experimental Paper. . . : . : . . . . . . 52

b. Theoretical Papers. . . . . . . . . . . 52

Part III: ALPHABETICAL LISTING OF AUTHORS . . . . . . 53 


\section{Part I: CHRONOLOGICAL LISTING}

\section{a. Experimental Papers}

1. L. M. Eisberg, W. B.' Fowler, R. M. Lea, W. D. Shephard, R. P. Shutt, A. M. Thorndyke, and W. L. Whittemore

Phys. Rev. 97, 797 (1955) (received Oct. 6, 1954)

$\pi^{-}$-p Interactions at $1.4 \mathrm{Bev}$

"... This indicated a slightly greater multiplicity than predicted by Fermi Siatistical Theory. Angle and momentum distributions of emitted pions are discussed in terms of possible pion-pion interactions and excited nucleun: slates, but conclusions on these questions arc uncertain."

2. R. Cool, O. Piccioni, and D. Clark

Phys. Rev. 103, 1082 (1956) (received Apr. 30, 1956)

Pion-Proton Total Cross Sections from 0.45 to 1.9 "Bev

"... A pronounced maximum for $\sigma\left(\pi^{-} \mathrm{p}\right)$ near. $0.9 \mathrm{Bev} .$. is confirmed

by data presented here.... The possibility of explaining this maximum with - resonant pion-pion.interaction as conjectured by Dyson and Takeda.is discussed."

Dygón quotes results of Piccioni et al., early experiments.

3. G. Maenchen, W. B. Fowler, W. M. Powell, and R. W. Wright

Phys. Rev.. 108, 350 (1957) (received July 10, 1957)

$\pi^{-}$- Proton Interactions at $5 \mathrm{Bev}$

"The presence of any resonant pion-nucleon or pion-pion interactions might be expected to yield $Q$ distributions of varying shapes. The similarity of all the $Q$ distributions indicates, therefore, that this experiment does not furnish evidence for resonant interactions between pairs of emitted particles."

(I think they may have a bump in $Q_{\pi}^{+}{ }_{\pi}^{-}$at $400 \mathrm{Mev}, \mathrm{M}_{2} \approx 4.9 \mathrm{~m}_{\pi^{*}}$ MLS)

4. W. D. : Walker

Phys. Rev. 108, 872 (1957) (received July 31, 1957)

$\pi^{-}$- Nucleon Interaction at $4.5 \mathrm{Bev}$

"The angular distributions are suggestive of a direct knockout of a pion by the incoming pion!"

5. Alberigi, Bernardini, Querzoli, Salvini, Silverman, and Stoppini (reported by G. Bernardini) .

Ninth Annual International Conference on High Energy Physics, Kiev, July 15-25, 1959, Vol: 1, 42 (1960)

Pion Photoproduction and Compton Effect on Nucleons

"They looked at. the possible production of the $\rho^{0}$ via the two-bodies reaction $\gamma+p \rightarrow p+\rho$, trying to detect the kinematically well-defined recoiling protons.... The authors consider that an upper limit to the heavy neutral meson cross section can be put safely ... as do/d $\Omega \leqslant 5 \times 10^{-3} \mathrm{~cm}^{2} / \mathrm{sr}$." 
6. G。 Goldhaber; W. B. Fowler, S. Goldhaber, T. F. Hoang, T. Kalogeropoulous, and $W$ 。 $M$ 。 Powell

Phys。Rev。 Letters 3, 181 (1959) (received July 17, 1959)

Pion-Pion Correlations in Antiproton Annihilation Events

"The experimental $Q^{2}$ distributions show no marked deviation from the

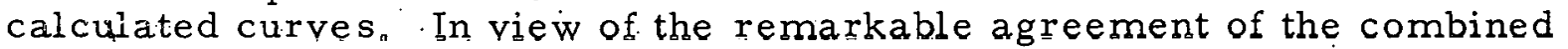
data with the LIPS (Lorentz Invariant Phase Space) model, we consider this model to be a good description of the overall model. We ascribe the deviations observed in like and unlike pion pairs to the presence of additional pion-pion correlations."

7. M. Blau, C。F.Carter, and A。 Perimutter

Nuovo cimento 14, 704 (1959) (received July 18, 1959)

Negative Pion Interactions at $1.3 \mathrm{Gev} / \mathrm{c}_{\mathrm{w}}$

$1 \%$. . the present data are not in disagreement with the calculated $\pi \cdot \pi$ interactions in so far as the latter are.valid."

8. W. Perkins III, J. Caris, R。 Kenney, and Vo Perez-Mendez

Phys.Rev。118, 1364 (1960) (received Oct。 7, 1959)

Pion Production by Pions

"... $\pi^{-}+\mathrm{p} \rightarrow \pi^{+}+\pi^{-}+\mathrm{n}_{\ldots} .$. total cross sections are $0.14 \pm .10 \mathrm{mb}$ at $260 \mathrm{Mev}, 0.71 \pm .17 \mathrm{mb}$ at $317 \mathrm{Mev}, 1.93 \pm .37 \mathrm{mb}$ at $37 \mathrm{l} \mathrm{Mev}$; and $3.36 \pm .74$ $\mathrm{mb}$ at $427 \mathrm{Mev}$. These results indicate a much larger cross section than the theoretical prediction based on the static model. Reasonable agreement can be obtained by the inclusion of a pion-pion interaction in the production mechanism."

9. V。 Go Zinov, $\Lambda$. D。 Konin, S. M。 Korenchenko, and B. Pontecorvo Soviet Phys."JETP 11, 1233 (1960) (reccived Jan。13, 1960)

A Search for the $\ddot{\rho}^{0} \overline{M e s o n}$ and a Check of Dispersion Relations in $\pi-N$ Scattering

"No anomalies in the energy dependence of the $\sigma_{t}^{-}$(total $\pi^{-}$-p cross section) were tound which could indicate the existance of a $\rho^{0}$ meson with $a$ : mass in the range 270 to $410 \mathrm{Mev}$."

10. I。 Derado

Nuovo cimento 15, 853 (1960) (received Feb。1, 1960)

Experimental Evidence for the Pion-Pion Interaction: at 1 Gev

"This result suggests that the maximum may occur. in the neighborhood of $60 \mathrm{Mev}$ in the $c_{0} \mathrm{~m}_{0} \mathrm{~s}$. If one assumes this: maximum to be real, then one can calculate the energy of the resonant $\pi-\pi$ state: $M_{\pi}=4.7 . m_{\pi^{*}} "$

11. G. Goldhaber, S. Goldhaber, W. Lee, and A. Pais

Phys. Rev。 120, 300 (1960) (received May 16, 1960)

Influence of $\overline{B o s}$-Einstein Statistics on the Antiproton-Proton Annihilation Process 
12: R. Gomez, H. Burkhardt, M. Daybell, H. Ruderman, M. Sands, and $R$. Talman

... Phys. Rev. Letters 5, 170 (1960) (recejved July 18, 1960)

Evidence Against the Existence of the B Meson

"This note reports an attempt to observe the production of such a meson in the reaction $\gamma+\mathrm{p} \rightarrow \mathrm{B}^{0}+\mathrm{p} \ldots$ The measurements yield an upper limit for the cross section."

\begin{tabular}{|c|c|c|c|}
\hline $\begin{array}{l}\mathrm{M}_{\mathrm{B}} \\
(\mathrm{Mev})\end{array}$ & $\begin{array}{l}\sigma_{\mathrm{c} \cdot \mathrm{m}^{2}}\left(\theta_{\mathrm{B}}\right) \\
\left(\mathrm{cm}^{2}\right)\end{array}$ & $\begin{array}{c}\theta_{B}\left(c \cdot m_{:}\right) \\
(\text {deg })\end{array}$ & $\begin{array}{l}\text { Energy of incident } \\
\text { photon } \\
\text { (Mev) }\end{array}$ \\
\hline 320 & $3.2 \times 10^{-32}$ & 40 & $690-1000$ \\
\hline 35.0 & 2.5 & 38 & $750-1000$ \\
\hline 380 & 2.7 & 36 & $750-1050$ \\
\hline 410 & 3.4 & 34 & $900-1100$ \\
\hline
\end{tabular}

13. E. Pickup, F. Ayer, and E. O. Salant

Phys. Rev. Letters 5, 161.(1960) (received July 27, 1960)

Single Pion Production in $0.96-\mathrm{Bev} \pi^{-}$-p Interactions

"No evidence of peaking in seen for $Q\left(\pi^{-} \pi^{+}\right)$, either for forward or backward neutrons, nor for $\left(\pi^{-} \pi^{0}\right)$ for forward protons. However, the $Q$ distribution for $\pi^{-} \pi^{0}$ in events with backward protons shows a distinct peak at about $325 \mathrm{Mev}$, suggestive of a $600-\mathrm{Mev}$ resonant "di-pion" state $\mathrm{T}=1$ or 2."

14. A. Abashian, N. Booth, and K. Crowe

Phys. Rev. Letters 5, 258 (1960) (received Aug. 11, 1960; revised Aug. 22, 19760)

Possible Anomaly in Meson Production in p+d Collisions

15. J. Button, P. Eberhard, G. R. Kalbfleisch, J. Lannutti, S. Limentani, $\therefore \quad \therefore$ G. Lynch, B. Maglić, M. L. Stevenson, and Nugyen-Xuong (as reported by F. Solmitz)

Proceedings of the 1960 Annual International Conference on High Energy Physics at Rochester (Conference.held Aug. 1-Sept. 1), page 166.

16. S. Goldhaber; G. Goldhaber, W. Powell, and R. Silberberg

Phys. Rev. 121; 1525 (1961) (received Sept. 1, 1960)

Antiproton Annihilations. in Propane.

"... study $\overline{\mathrm{p}}$ annihilation at' $\mathrm{l} .05 \mathrm{Bev} / \mathrm{c}$. It was observed that $\mathrm{K}-\mathrm{mes}$ on production rises sharply with increase in energy'from $4 \pm 1 \%, \bar{p}$ at rest to $8 \pm 1 \% .$. the pion multiplicity $(<n>=5.0 \pm .2)$ was not observed to increase appreciably with... energy. it.

17. N.E. Booth, O. Chamberlain, and E. H. Rogers

Nuovo cimento 19, 853 (1961) (received Oct. 10, 1960)

Search for a Neutral Meson of: Zero I-Spin

18. T.: F. Hoang, Phys. Rev. 121, 1523 (1961) (received Aug. 22, 1960; revised manuscript received Nov. 17, 1960)

Analysis of $\mathrm{K}-\mathrm{Mes}$ on Production by $\overline{\mathrm{p}}$ Annihilation 
${ }^{9} \mathrm{~K}-\mathrm{Meson}$-production by $\overline{\mathrm{p}}$ annihilation has been investigated using an isobar model. A comparison of the predictions of this model with the experimental data excludes the assumption of an isobar state of $(\pi \pi)$ having a mass greater than three pion masses.

19. J.'G. Rushbrooke and D. Radojüić

Phys. Rev. Letters 5, 567.(1960) (received Nov. 21, 1960)

Search for Resonance in $\pi-\pi$ Interaction in $\pi-N$ Scattering at $0.96 \mathrm{Bev}$

Reaction $\pi^{-}-p \rightarrow p+\pi^{-}+\pi^{0}$. "A strong peak at $-\omega^{2} / \mu^{2}$ is evident in the spectrum of all our events.". ( $\left.\mathrm{M}_{\pi} ; \doteq 650 \mathrm{Mev}.\right)$

20. J. A. Anderson, V. Bang, P. Burke, D. Carmony, and N. Schmitz Revs. Mode-rn Phys. 33, 431 (1960) (received Dec. 27, 1960)

Pion-Pion Interactions

21. N. E. Booth, A. Abashian, and K. M. Crowe

Revs. Modern Phys. 33, 393 (1961) (recuived Dec. 27, 1960)

Double Mèson Production in Proton-Deuteron Collicions

22. G. Goldhaber and W. Lee

Revs. Modern Phys. 33, 402 (1961) (received Dec. 27, 1960)

$\pi-\pi$ Correlations. in $\overline{\mathrm{p}}-\mathrm{p}$ Annihilation

$\begin{array}{lll}\text { Number of pion pairs } & \begin{array}{c}\gamma \operatorname{expt} N\left(\cos \theta_{\pi \pi}>0\right) \\ \equiv\left(\cos \theta_{\pi \pi}<0\right)\end{array} \\ \text { like } & 752 & 1.23 \pm .10 \\ \text { unlike } 1504 & 2.18 \pm .12\end{array}$

23. E. O. Salant, E. Pickup, D. K. Kobınsont, and B. A. Munir

Rev.s. Modern Phys. 33, 435 (1961) (received Dec. 27, 1960)

Pion-Pion Interactions and Pion Production Branching Ratios at the Third Resonance

24: B. A. Munir, E. Pickup, D. K. Robinson, and E. O. Salant

Phys. Rev. Letters, 6, 192 (1961) (received Jan. 30, 1961)

Pion Production Branching Ratios Through the 900-Mev Resonance.

25. K. Berkleman, G. Cortellessa, and A. Reale

Phys. Rev. Letters 6, 234 (1961) (received Feb. 8, 1961)

Search for the $\omega$ in Photo Production

"The $\omega\left(\rightarrow \pi^{0}+\gamma\right)$ photo production yield in the present experiment is

less than. $1 / 30$ of the $\pi$ yield at the same energy and angle and is certainly small compared with the direct double-pion yield."

26. D。 N. Olson, H. F. Schopper, and R. R. Wilson

Phys. Rev。 Letters 6, 286 (1961) (received Feb. 15, 1.961)

Electromagnctic Properties of the Proton and Neutron" 
27. R. Hofstadter and $R$. Herman

Phys. Rev。 Letters 6, 293 (1961) (received Feb. 15, 1961) Electric and Magnetic Structure of the Proton and Neutron

28. R. Hofstadter, Co Devries, and Robert Herman Phys. Rev. Letters 6, 290.(1961) (received Feb. 15, 1961) Dirac and Pauli Form Factors of the Neutron

29. J. Anderson, V. Bang, P. Burke, D. Carmony, and N. Schmitz Phys. Rev. Letters 6, 365 (1961) (received March 2, 1961) Experimental Cross Section on the $\pi-\pi$ Interaction

"Our data do show a rise in the $\pi-\pi$ cross section starting at $\omega^{2}=17$, reaching a value of the order of $200 \mathrm{mb}$ at $\omega=20$ to $22 \ldots$ However, in this region of $\omega^{2}$ our extrapolation distance begins to get larger, making the extrapolation procedüe less conclusive."

30. Y. A. Batusov, S. A. Bunyatov, V. M. Sidorov, and V. A. Yarra

Zhur. Eksptl.i Teoret. Fiz。40, 1528 (1961); Sov。 Phys. JETP 13, 1070 (1961) (received March 16, 1961)

Ratio of $\pi N \rightarrow \pi \pi N$ Reaction Cross Sections of $290 \mathrm{Mev}$ and $\pi-\pi$ Interaction

"The data of the present work contradict the as sumption that there exists a resonance in the interaction of two $\pi$ mesons at a total energy of $310 \pm 10 \mathrm{Mev}$ in a $\mathrm{T}_{\pi}=1$ state. The interaction in $\mathrm{T}_{\pi}=0$ state seems to dominate."

31. Y. K. Akimov, V. I. Knmarov, K. S. Marish, O. V。 Savchenko, and L. M. Soroko

Zhur. Eksptl, i Teoret. Fiz。40, 1532 (1961); Soviet Phys. JETP 13, 1073 (1961) (received March 22, 1961)

Search for Anomalies in the Spectrum of the $\mathrm{H}^{3}$ Nuclei Emitted in the Reaction $\mathrm{p}+\mathrm{d} \rightarrow \mathrm{H}^{3}+\pi+\pi$ at a Proton Energy of $670 \mathrm{Mev}$

11... The results obtained here do not confirm the existence of a resonance of two $\pi$ mesons in the $T=1$ state and total energy from 275 to $420 \mathrm{Mev}$ and also allow us to exclude the existence of $\omega$ particle with $\mathrm{T}=1$ and Mass $\approx .310 \mathrm{Mev}_{\text {.". }}$

32. D. Robinson, B. Munir, E. Pickup, and E. Salant

Bull. Am. Phys. Soc。6, 301 (1961); Meeting of American Physical Society, Washingtón, April 24-27, 1961

Single Pion Production in $\pi^{-}$-Proton Collisions at 0.9 and $1.1 \mathrm{Bev}$

"The momentum distributions of the protons and the neutrons at both energies ( $1.1 \mathrm{Bev} \pi^{-}$and $0.9 \mathrm{Bev} \pi^{-}$) have low energy groups in disagreement with the predictions of the statistical and standard isobar theories of pion production. The significance of these results with reference to nucleon isobar and pion-pion interactions will be discussed."

33. A. R. Erwin, R. March, W. D. Walker, and E. West

Phys. Rev。 Letters 6, 628 (1961) (received May 11, 1961)

Evidence for a $\pi-\pi$ Resonance in the $I=1, J=1$ State

"The results indicate a peak in the neighborhood of $750 \mathrm{Mev}$ with a width of $150-2 ; 00 \mathrm{Mev}$, which is about $3 / 4$ of what it would be $\left(12 \pi \lambda^{2}\right)$ 
for a resonance in the $\mathrm{I}=1, \mathrm{~J}=1$ state."

34. W. Walker, H. Fechter; R. March, D. Lyon, P. Satterblom, and A. Erwin

Bull.Am Phys.Soc. 6, 311 (1961)

$\pi-\mathrm{p}$ Interactions at $1 . \overline{97} \mathrm{Bev} / \mathrm{c}$

35. D. Stonehill, C. Baltay, H. Courant, W. Fickinger, E. C. Fowler

H. Kraybill, J. Sandweiss; J. Sanford, and H. Taft

Phys. Rev. Letters 6, 624 (1961) (received May 12, 1961)

Pion-Pion Interaction in Pion Production by $\pi^{+}-p$ Collisions

"... If charge independence is assumed, a resonance in $I=2$ state should appear $80 \%$ as $n \pi \pi^{7}$ reactions and $20 \% p \pi \pi$. We conclude that the interaction responsible for the observed peaks is overwhelmingly in the state $I=1$, and possibly not at all in the $I=2 \ldots$ the data are not inconsistent with a single resonance with $\omega$ between 5.0 and 5.5 , corresponding to a total dipion mass of 700 to $770 \mathrm{Mev}$. The full width a.t half maximum appears to be $\approx 90 \mathrm{Mev}$. (The rms error in the determination of $Q$ values is less than 10 Mev.)!"

36. N. E. Booth, A. Abashian, and $\mathrm{K}$. Crowe Phys.: Rev. Letters 7, 35 (1961) (received June.12, 1961) Anomaly in Meson Production in. p + d Collisions

37. R. M, Littauer, H. F. Schopper, and R. R. Wilson Phys. Rev。 Letters 7, 141 (1961) (received July 21, 1961) Scattcring of Bev Electrons by Hydrogen and Deuterium

38. R。 M. Littauer, $H_{0}$. F. Schopper, and $R$. R。 Wilson Phys。Rev。Lettcrs 7, 144 (1961) (received July 21, 1961) Structure of the Protori and Neutron

39. B.C. Maglic, G. R. Kalbfleisch, and M. L. Stevenson Phys. Rev。Letters 7, 137 (received July 27, 1961) Charge Asymmetrie $\bar{s}$ in the Angular Distribution of $\pi$ and $\mathrm{K}$ Mesons from Antiproton Annihilations in Flight

"It will not be possible, however, to obtain a quantitative answer as to the importance of the Bose effect and/or $\pi-\pi$ interaction without further extensive studies."

40. M。 Ferro-Luzzi, D. H. Miller, J. J. Murray, A. H. Rosenfeld, and R. D. Tripp

Nuovo cimento (to be published) (UCRL-9798, Aug. 4, 1961) Analysis of $1347 \tau^{-}$Decays

41. B. Magli6, L. W. Alvarez, A. H. Rosenfeld, and M. L. Stevenson Phys. Rev. Letters.7, 178 (1961) (received Aug. 14, 1961) Evidence for a $\mathrm{T}=0$ Three-Pion Resonance 
42. E. Pickup, D。 K. Robinson, and E。 O. Salant

Phys. Rev。 Letters 7, 192 (1961) (received Aug. 14, 1961)

$\pi-\pi$ Resonance in $\pi^{-}-\mathrm{p}$ Interactions at $1.25 \mathrm{Bev}$

"We obtain $\sigma_{1-\pi}=95 \mathrm{mb}$ at the peak, $\omega^{2}=29 \mu^{2}(\mathrm{Q}=475 \pm 10 \mathrm{Mev}$,"

$M=755 \mathrm{Mev})$. $\pi-\pi$ The full width at half $\max$ is $130 \mathrm{Mev}$. These.values and the values determined at 1. 74 Bev by Erwin et al. (Ref. 33) agree within statistics (the Adair analysis) is suggestive of $\mathrm{J}=1$ rather than larger $\mathrm{J}$ values which would give higher powers of $\cos ^{2} a$."

43. A. Pevsner, K。 Kraemer, M。 Nussbaum, P. Schlein, M. Block, A. Kovacs, and C. Meltzer

(Preprint received by compiler Sept, 12, 1961)

Three-Pion Resonances in $\pi^{+}+d$ Reactions

44. M. L: Stevenson, L。 W。 Alvarez, B. C. Maglic̀, and A. H. Rosenfeld Phys。Rev。 Jan。15, 1962 (UCRL-9856, Sept, 15, 1961)

Spin and Parity of the $\omega$ Meson

45. N. Xuong and G。 R. Lynch

Phys.Rev. Letters 7, 327 (1961) (received Sept. 15; 1961)

Evidence Confirming the $\mathrm{T}=0$ Three-Pion Resonance

46. Yu。K。Akimov, V。I.Komarov, K. S. Marish, O.V. Savchenko, and $L_{0}$. M. Soroko

Dubna Report D-714, 1961

$\pi-\pi$ Anomalies of the $\mathrm{H}^{3}$-Spectrum in Reaction $\mathrm{p}+\mathrm{d} \rightarrow \mathrm{H}^{3}+\pi^{+}+\pi^{0}$ at the Proton Energy of $670 \mathrm{Mev}$

11. The results obtained in these experiments do not confirm the existence of the $\pi-\pi$ resonance in the states with $T=1$ for the interval of the total energy in $c_{0} \mathrm{~m}$. $\mathrm{s}$. of two mesons from $\pi \pi 275$ up to $400 \mathrm{Mev}$. They allow also to conclude that there exists no $\omega$ particle with $T=1$ and the mass of $310 \mathrm{Mev}$.

32. Comparison of the reactions $\mathrm{p}+\mathrm{d} \rightarrow \mathrm{H}^{3}+\pi^{+}+\pi^{0}$ and $\mathrm{p}+\mathrm{d} \rightarrow$

$\mathrm{He}^{3}+\pi+\pi$ shows that the $\sigma$ for two-pion production in $\mathrm{T}=0$ exceeds approximately by an order of magnitude that with $\mathrm{T}_{\pi \pi}=1 \pi \pi_{\mathrm{p}}$ to $\mathrm{M}_{\pi \pi}=400 \mathrm{Mev}$."

47. R. Barlontaud, C. Choquet, C. Gensollen, J.Heughebaert, A. Leveque, $\mathrm{J}$. Meyer, and $\mathrm{G}_{\text {。 }}$ Viale

Presented at the International Conference on Elementary Particles at Aix-en-Provence, France ('received by compiler Sept. 14, 1961)

Pion Production by Positive Pions at 820 and $900 \mathrm{Mev}$

48. J. Kirz, j。 Schwartz, and R. Tripp

Submitted to Phys.Rev。(UCRL-9935, Nov。 6, 1961)

$\mathrm{I}=2 \mathrm{~S}-$ Wave Pion-Pion Scattering Length

"We found that the cross section for the reaction $\pi^{+}+\mathrm{p} \rightarrow \pi^{+}+\pi^{+}+\mathrm{n}$

(at $475 \mathrm{Mev} / \mathrm{c}$ ) is $0.12 \pm .01 \mathrm{mb}$. A Chew-.Low extrapolation gives

$a_{2} \leqslant 0.15 \lambda_{\pi}$ for the $I=2$ scattering length." 


\section{b. Theoretical Papers}

49. I. I. Pomeranchuk

Doklady Akad. Nauk S. S.S.R. 78, 889 (1951)

50. O. Picrinni

Third Annual Rochester Conference on High Energy Physics, December, 1952

Private communication on $\pi-\pi$ interaction

See also Dyson, Ref., 56, and Kovacs, Ref. 51.

51. J. S. Kovacs

Phys. Rev. 93, 252 (1954) (received Nov. 6, 1953)

The Angular Correlation of Mesons Produced in Inelastic Meson-Nucleon Collisions

"In high-energy meson-nucleon.collisions in which two mesons appear in the final state, any appreciable...correlation in the angle between outgoing mesons. will be strongly dependent upon the relative strengths of the mesonnucleon and meson-meson coupling. "(He thanks Piccioni for stimulating the work.)

52. A. N. Mitra and F. J. Dyson

Phys. Rev。 90, 372 (1953) (received Jan. 22, 1953)

Evidence for Direct Meson-Meson Interaction

"Our calculations confirm that no long-range forces are predicted by meson theory except as the result of a direct meson-meson interaction, thus giving the possibility of obtaining this interaction from the experiments. "

53. Marc Ross

Phys. Rev。 95, 1687 (1954) (received July 30," 1954)

Meson-Meson Interaction in Meson Scattering

"It shall be assumed that the meson-nucleon scattering arises from two sources: $A$, the meson-nucleon interaction neglecting any meson-meson collisions... and $B$, meson interaction direclly. with the mesons of the. nucleons proper field."

54. H. Miyazawa

Phys. Rev. 97, 1399 (1955). (received Nov. 22, 1954)

Strong-Coupling Theory for the Case of Pseudoscalar Coupling

"... A strong-coupling approximation is employed to diagonalize the Hamiltonian at each point. The vacuum is represented by a set of rotators each having excited states. Propagation of this excitation through the lattice points has the character of particles which are identified as pions and excited states of pions." 
55. M. Ho Johnson and.E. Tejler

Phys. Rev。 98, 783 (1955) (received Jan. 26, 1955)

Field Theory of Nuclear Forces

56: F'. J. Dysion

Phys. Rev: 99, 1037 (1955) (received May 23, 1955)

Second Maximum in the Negative Pion Scattering Cross Section

Interprets the early nie asurements of $\pi^{-}-p$ and $\pi^{+}-p$ total cross section as possible evidence for a resonant state of two pions. "( This idea was suggested by $O$. Piccioni.several years ago, before any experiments existed to support it.)."

Dyson reasoned that since the resonance didn't show up in the total cross section for $\pi^{+} \mathrm{p}$, it was evidence for $\pi^{*}$ to be $I=0,2$ ( since $\pi^{+} \mathrm{p}$ doesn't. have its $\pi^{* * *}$ in $[=0.1$

57. D. Ito and S. Minami

Progr。Theoret. Phys。(Kyoto) 14, 482 (1955) (received July 20, 1955)

On the Angular Distribution of Double Pion Production in Pion-Nucleon Collisions at $1.4 \mathrm{Bev}$

"If we assume that pion-pion interaction plays a more important role as is done by Kovacs, both postulates I and II are fulfilled."

58. G. Takeda

Phys. Rev。 100, 440 (1955) (received Aug.9, 1955)

Strong Pion-Pion Interaction Model Applied to Pion-Nucleon Scattering in $1-\mathrm{Bev}$ Region

As sumptions:

(1) Nucleon a compound. system with one pion in cloud.

(2) Strong interaction between incident $\pi$ and cloud pion.

(3) Incident pion and nucleon core don't interact.

Prediction: $\mathrm{T}=1, \mathrm{~J}=1$ 。

59. S. Z. Belenky and L. D. Landau

Uspekhi Fiz. Nauk.56, 309 (1955) and Nuovo cimento 3, Supplement 15 (1956)

Hydrodynamical Theory of the Multiple Production of Particles

Following criticism of Fermi model:

"Some 'of' his (Fermi) assumptions and quantitative calculations are not convincing. Thus, his assertion that the number of particles in a nuclear star. is determined by the number of particles created in the volume $V$ at the moment of collision is unfounded. Owing to high density of the particles and to strong interaction between them, one.cannot really speak of their number at that moment. Actually the system expands and the number of particles becomes definite only when the interaction between them becomes small(!!) This has been pointed out by. Pomeranchuk (Doklady Akad. Nauk. S.S.S. R. 78, 889 (1951)." :

60. H. Duerr and E. Teller

Phys. Rev。101,495 (1955) (received Nov, 9, 1955)

Interaction of Antiprotons with Nuclear Fields 
61. S. Z. Belenky

Nuclear Phys. 2, 259 (1956). (received July 17, 1956)

Connection Between Scattering and Multiple Production of Particles

"According to Fermi 's theory, multiple production of particles in high energy collisions should be determined by the statistical weight of the respective state. In the present investigation the statistical weight is calculated with account of mutual interaction of pairs of paiticles and it is ohown that in this case the scattering phase of the interacting particles is involved as a characteristic of the interaction. In resonance scattering the effect of the interaction reduces to the appearance of an intermediate "isobaric". state which would be included in the statistical theory. " Beautiful paper!! Landau example: Partition functions: $Z_{1}$, discrete states; $Z_{2}$ continuous states:

$$
z=z_{1}+z_{2} ; z_{2}=1 / \pi \int_{0}^{\infty}(2 \ell+1) \frac{\partial \eta_{\ell}}{\partial p} \exp \left(-p^{2} / m T\right) d p
$$

where $\eta_{l}(p)=$ scattering phase.

For resonances $\eta_{l}=\eta_{l}^{0}+\arctan \frac{\Gamma}{E_{0}-E}, z_{2}=(2 l+1) \exp \left(-p_{0}^{2} / \mathrm{mT}\right.$,

where $\eta_{l}^{0}$ is a nonresonant phase. In effect, $\eta_{\ell}$ acts just like a discrete particle.

62. E。 Teller

Proceedings of Sixth Annual Conference on High-Energy Nuclear Physics,

Rochester (Conference held Apr. 3-7, 1956), p. VII - 18:

Proposed Explanation of Strong Antinucleon-Nucleus. Interaction

63. I. Dyatlov, V. Sudakov, and K. Ter-Martirosyan

Snviet Phys.JETP 5,631 (1957) (received Dec. 18, 1955)

Asymptotic Meson-Meson Scattering Theory

64. G. Sudarshan

Phys. Rev, 103, 777 (1956) (received Feb. 27, 1956)

Annihilation of Antinucleons

"In this note, the expected $\pi^{-}$and $\mathrm{K}$-meson multiplications resulting from the annihilation of a nucleon pair at rest have been calculated on the basis of two versions of the statistical theory, the Fermi and the Pomeranchuk-Landau models. The Fermi model yields smaller.pion multiplicities and larger probabilities for $K$-meson pairs than does the Pomeranchuk-Landau model. Volume of interaction should be replaced by $\Omega^{\beta}=\mathrm{n} \Omega$, where $\Omega$, is effective volume of interaction for a single pion. This then introduces an additional factor $(n)$ into the statistical factor. This weights higher multiplicities."

65. $\mathrm{H}_{0} \cdot$ Duerr

Phys。Rev.103, 469 (1956) (received March 30, 1956)

Relativistic Effects in Nuclear Forces 
66." S. Z。 Belenkii

Zhur. Eksptl.i Teoret. Fiz. 1171 (1957); Soviet Phys。JETP 5, 952

(1957) (received July 5, 1956)

Relation Between Scattering and Multiple Particle Production

"... In resonance scattering, the effect of the scattering reduces to the appearance of an intermediate' 'isobaric' state which should be included in the statistical theory."

67. A. N. Mitra and R。P.Saxena

Phys: Rev. 108, 1083 (1957) (received Feb.25, 1957)

Meson-Meson Interaction in the Bethe-Salpeter Approximation

68. Y. Nambu

Phys. Rev。106, 1366 (1957) (received April 25, 1957)

Possible Existence of a Heavy Neutral Meson

in an attempt to account for the charge distributions of the proton and neutron as indicated by the electron scattering experiments we would like to consider the possibility that there may be a heavy neutral meson which can contribute to the form factor of the nucleon. We assume this meson, $\rho^{0}$, is a vector field with isotopic spin zero and a mass two to three times that of the ordinary pion, coupled strongly to the nucleon field. It should be added that the neutral meson considered here is similar in nature to the one introduced by Teller for quite different purposes (Refs. 55, $60,62,64)$.

69. T. Goto

Nuovo cimento 8, 625 (1958) (received Oct.9, 1957)

Pion-Pion Interaction and Multiple Pion Production in Nucleon - Anti nucleon Annihilation Process

"The multiple pion production in antinucleon-nucleon annihilation process is investigated by means of the statistical theory with the strong pion. pion interaction. The characteristics of the isobar state of two pions are determined from the observed momentum distribution of a nucleon in the reaction $\pi^{-}+p \rightarrow N+2 \pi$ at 1 Gev or from the theories of Takeda, Dyson, and Miyazawa."

G. Takeda (Ref。 61 and F。Dyson (Ref。 56) proposed $\pi \cdot \pi$ interaction to explain the "second maximum" in $\sigma_{\text {total }}(\pi \mathrm{p})$.

"... One of the most elegant methods that included the effects of final interactions in Fermi's statistical theory was proposed by Belenkii (Nuclear Phys. 2, 259. (1956)。" By looking at normal spectrum of proton in $\pi^{-}+\mathrm{p} \rightarrow \mathrm{N}+\pi^{-}+\pi^{+}$he predicts $\mathrm{Q}_{\pi \pi}=350$ to $400 \mathrm{Mev}$ or $\mathrm{M}_{\pi \pi} \approx 670$. Makes specific reference to $\bar{p}+p \rightarrow 3 \pi$. $\pi \pi$ Quite a paper?

70. G. F. Chew; Robert Karplus, S. Gasiorowicz, and F。 Zachariasen Phys. Rev. 110, 265 (1958) (received Nov. 25, 1957)

Electromagnetic Structure of the Nucleon in Local-Field Theory

"It is argued that the main contributor to the magnetic moment structure in the spectral distribution must be the two-pion state, and an attempt is made to evaluate this contribution in terms of the known behavior of pion- 
nucleon scattering. It is emphasized that the large observed charge radius of the proton does not imply the dominance of the two-pion state in the charge structure. Thus it is not impossible that higher mass configurations supply the isotopic scalar charge needed to explain the small neutron-electron interaction.

71. ए。 Eberlc

Nuovo cimento 8, 610 (1958) (received March 26, 1958)

Pion Multiplicity in Antinucleon Annihilation

"Pion multiplicity in antinucleon annihilation has been calculated using Dyson's hypothesis of pion-pion resonance interaction $(T=0)$. The percentage of $\mathrm{K}$ mesons was also evaluated supposing that their volume of interaction has a radius given by the Compton wave length of $K_{2}$ mesons. Results are in reasonable agreement with experimental data."

$$
\begin{aligned}
& S\left(n_{\pi}\right)=K_{n, T}\left[S_{0}\left(n_{\pi}\right)+K,(2 l+1) S_{0}(n-2), 1 W_{0}\right)+K_{2}(2 l+1)^{2} S_{0}((n-4) \\
& \left.2 W_{0}\right)+\ldots . .
\end{aligned}
$$

$K$ is the coefficient that takes into account the conservation of total isơtópic spin; the various $K, \mathrm{~T}$ have been calculated by Yeivin and DeShalit. contained

The statistical weights $n, T$ have to be calculated as if the final 'state n pions,

$(\mathrm{n}-2)$ pions plus a "particle" $\mathrm{W}$

$(\mathrm{n}-4)$ pions plus two "particles ${ }^{\circ \mathrm{P}^{\prime}} \mathrm{W}_{0}$,

and so on.

72. S, No Gupta

Phys。Rev. 111,1698 (1958) (received May 19, 1958)

Pion Production in High-Energy Processes

"Tt. is shown that the high-energy experiments on pion production favor rather than rule out the possibility of the existence of a new highly unstable neytral scalar meson (the $\rho^{0}$ meson)" ...the $\rho^{0}$ meson can decay only as $2 \pi^{0}$ or $\pi^{+}+\pi^{-}$.

73. P. Federbush; M. L。 Goldberger, and S。 B. Treiman

Phys. Rev。 112, 642 (1958) (received June 6, 1958)

Electromagnetic Structure of the Nucleon

(Only remarks relevent to $3 \pi$ are emphasized here)

$" \because \therefore$ The least massive state which contributes to the isotopic scalar properties of nucleons is the three-pion state; and one would expect this state to be the most important one in determining the isotopic scalar magnetic moment and the mean square radii.

$$
\begin{aligned}
& <0|j| p^{+} p^{-} p^{0} \text { out }>=2 E(\underset{m}{(k q})\left(8 w_{+} w_{-} w_{0}\right)^{-1 / 2} H^{*}\left(k^{2}, q^{2}, \lambda^{2}\right) \\
& \lambda^{2}=\mathrm{k} \circ \mathrm{q} /|\mathrm{k}| \mathrm{g}|\mathrm{q}| \stackrel{\mathrm{k}}{+} \stackrel{\mathrm{q}}{\longrightarrow} 0
\end{aligned}
$$

This matrix element transforms like a psuedo (axial) vector. 
"In conclusion, there remain at least the following four alternatives: (1) The experiments are wrong and the proton charge radius is very small (this would obviously be the nicest solution for theoreticians). (2) The two-pion state is grossly mistreated in our theory so that one gets a large vector charge radius, and necessary isotopic scalar quantities ultimately appear, presumably from the three-pion state. (3) States of uncomputable complexity are important. (4) Our whole dispersion approach is wrong. The last possibility would be catastrophic.".

74. V. I. Rus'kin

Zhur. Eksptl。i Teoret Fiz。 36, 164 (1959); Soviet Phys. JETP 9, 113 (1959) (received June 14, 1958)

Effects of a Resonant Pion-Pion Interaction in Fermi's Statistical Theory of Multiple Particle Protirction

"The statistical theory of Fermi is applied to $\pi^{-}-\mathrm{p}$ scattering at 4.5 Bev, including effects of a possiblc resonant interaction between two pions."

75. R。 W. Huff

Phys。Rev。112, 102\.(1958) (received June 16, 1958)

Neutral Vector Meson and Nucleon Form Factors and Magnetic Moments

"The existence of the heavy neutral vector meson recently suggested by Nambu is studied as a possible explanation of two phenomena, (1) the small charge root-mean-square radius of the neutron, $\because$ and (2) the nucleon anomalous magnetic moments."

76. S. D。 Drell

Proceedings of 1958 Annual International Conference. on High-Energy Physics at CERN (June 30, 1958), pp. 20-33

Nucleon Structure

77. C. Goebel

Phys. Rev. Letters 1, 337 (1958) (received Aug。25, 1958) Determination of the $\pi-\pi$ Interaction Strength from $\pi-N$ Scattering

"Among the effects which can be qualitatively argued to be a result of the $\pi-\pi$ interaction are the following:

(a) nònresonant behavior of $i=1 / 2 \mathrm{~s}$-wave phase shift at low energy...;

(b) the positive value of $\delta$ above $\approx .200 \mathrm{Mev}$ and the positive rise of the $i=1 / 2$ scattering amplitude in the region 200 to $500 \mathrm{Mev}$;

$\left(b^{p}\right)$ the large value of the meson production cross section near threshold in the $i=1 / 2$ state but not in the $i=3 / 2$;

(c) the maximum of the total cross section in the $i=1 / 2$ state at $\approx 0.9$ Bev (the "second" maximum);

(d) the large size of the high-energy total cross section, and the subsequent backward peaking of the nucleon's angular distribution in both elastic and inelastic events of low multiplicity."

78. L. B. Okun' and I. Y。 Pomeranchuk

Zhur. Eksptl。i Teoret. Fiz. 36, 300 (1959); Soviet Phys。JETP 9 , 207 (1959) (received Sept.20, 1958)

- Peripheral Interactions between Elementary Particles 
79. Y: Fujii

Progr. Theoret. Phys. (Kyoto) 21, 232 (1959) (received Oct. 9, 1958)

On the Analogy Between Strong Interactions and Electromagnetic Interaction

"According to Sakata's composite hypothesis of heavy particles, we assume that the fundamental particles do not change their kind through strong.interaction... If we assume that the phase function depends on the space-time coordinate, it leads to the existence of a neutral vector meson analogous to the electromagnetic field, the mass of which need not always vanish. Its consequences are examined and found to be quite favorable for the interpretation of the strorg interaction. In particular, it:leads to the conservation law of parity in strong interactions and the condition for the existence of the composite state."

80. G. F. Chew and F.E. Low

Phys. Rev。113, 1640 (1959) (received Nov. 3, 1958)

Unstable Particles as Targets in Scattering Experiments

"... The method is useful for unstable particles from which free targets cannot be made; the special examples of pion and neutron targets are discussed in detail."

81. S. N. Gupta

Phys. Rev. 111, 1436 (1958) (received April 28, 1958) also Phys. Rev. Letters 2, 124 (1959) (reccived Dec. 19, 1958)

Effect of Strange Particles on Magnetic Moments of Nucleons; Theory of Spin-Orbit Interaction in Nuclear Forces

"The effect of heavy mesons on the anomalous magnetic moments of nucleons is discussed. The present investigation suggests that (a) $\mathrm{K}$ mesons behave as pseudoscalar particles in strong interactions, and (b) there exists a hitherto unobserved highly unstable heavy neutral scalar meson, which is coupled strongly to the nucleons."

82. Ko ishida.

Progr. Theoret. Phys. (Kyoto) 25, 797 (1961) (received Jan. 11, 1959) The Effect of the Pion-Pion Interaction on the Pion-Nucleon Scattering

'It is suggested that the low-energy pion-nucleon $\mathrm{p}$-wave phase shifts will be well understood by taking into account the effects of not only the pion-pion $\mathrm{P}$-wave interaction but also the pion-pion $\mathrm{S}$-wave interaction into the equations for the $\mathrm{p}$-wave scattering amplitudes derived by Chew, Goldberger, Low, and Nambu."

83. A. A. Ansel' $m$ and,V. N. Gribov

Zhur. Eksptl. 1. Teoret. Fiz.36, 1890 (1959) (received Jan. 19, 1959); Soviet Phys. JETP 9, 1345 (1959)

On the Possibility of Determining the $\pi-\pi$ Scattering Amplitudes from the Analysis of the $y+p \rightarrow N+\pi+\pi$ Reactions Near Threshold

"It is shown that a study of $\gamma+p \rightarrow p+\pi^{+}+\pi^{-}, p+\pi^{0}+\pi^{0}, n+\pi^{+}+\pi^{0}$ rear threshold allows the letermination of the $\pi-\pi$ zero-energy scattering amplitudes if the $\pi-\pi$ interaction has resorance character, and the determination of a certain combination of these amplitudes if the interaction does rot have resonance character." 
84. B. Bosco and V. De Alfaro

Phys. Rev. 115, 215 (1959) (received Feb. 2, 1959)

Three-Pion Contribution to the Electromagnetic Structure of the Nucleon

85. A. A. Ansel' and V. N. Gribov

Zhur. Eksptl。i. Teoret。 Fiz。 37, 501, (1959); Soviet Phys. JETP 10, 354 (1960) (received March 9, 1959)

On the Possibility of Determining the Amplitude for Charge-Exchange Pion-Pion Scattering from an Aralysis of the $\pi^{-}+\mathrm{p} \rightarrow \mathrm{N}+\pi^{+}+\pi^{-}$ Reaction Near Threshold

86:" W: R. Frazer and J。 R. Fulco

Phys. Rev。 Letters 2, 365 (1959) (received March 25, 1959)

Effect of a Pion-Pion Scattering Resonance on Nucleon Structure:

(See also Ref。 107。)

87. V. I. Rus'kin

Zhur. Eksptl。i Teoret. Fiz。37, 105 (1959); Soviet Phys. JETP 10 ,

74 (1960). (received March 31, 1.959)

$\pi^{-}-\mathrm{p}$ Interaction at $1.4 \mathrm{Bev}$

"The statistical theory of Fermi, taking into account the resonance between a pion and a nucleon as the resonance interaction between two $\pi$ ' mesons, is used to explain the experimental results of the $\pi \circ p$ interaction at $1.4 \mathrm{Bev}$."

88.. B. S. Thomas and $W$. G. Holladay

Phys. Rev。 115, 1329 (1958) (received April 6, 1959)

Pion-Pion Interaction in $\tau^{+}$Meson Decay

89. V. S. Barashenkov and V. M. Maltsev

Zhur. Eksptl.i Teoret。Fiz。37, 884-(1959); Soviet Phys. JETP 10, 630 (1960) (received $\overline{\text { May }} 20,1959)$.

Resonance Interaction of Pions

"... available experimental data can, within the limits of experimental error, be explained without employing the hypothesis of resonance. $\pi-\pi$ interaction."

90. D. Ito, M. Yamazaki, T.Kobayàshi, and K. Mori

Progr. Theor. Phys. (Kyoto) 22, 448 (1959) (received May 28, "1959)

Pion Production in Pion-Nucleon Collision with Assumption of Strong

Pion-Pion Interaction

"We have only tried the simplest type of pion-pion interaction; therefore, we cannot conclude anything definite about the evidence of strong pion-pion interaction." "? 
91. Y. Yamaguchi

Progr。 Theoret. Phys. (Kyoto) Suppl. 11, (1959) (received June 17, 1959) A Composite Theory of Elementary Particles

"A composite theory of particles is investigated, based on six fundamental particles ( $\mathrm{p}, \mathrm{n}, \Lambda, \nu, \mathrm{e}$, and $\mu$ ). We assume three types of interaction, very strong (VSI), moderately strong.(MSI), and weak (WI) interaction, besides electromagnetic couplings. The VSI is global (i.e., completely symmetrical with respect to $p, n$, and $\Lambda$ ) and gives rise to major parts of baryonic mass but is missing among the leptons. This is why leptons are so light. This VSI is also responsible for creation of various bound states, pions, kaons, etc. from baryon-antibaryon pairs. If there were only VSI, masses of $n, p, \Lambda$ and those of pion and kaon would be equal. The charge-independent MSI splits the mass degeneracies between nucleons and $\Lambda$, pions and kaons, etc. and also $(e, v)$ and muon. We can conclude that the kaon is pseudoscalar. and the $\Lambda-\Sigma$ and $\Xi-N$ relative parity must be odd, where the $\Sigma$ or $\Xi$ is the bound state of $\Lambda+\bar{N}+N$ or $\Lambda+\bar{N}+\Lambda$. There are open possibilities of existence of baryons and mesons with higher values of strangeness. The Feynman-Gell-Mann Theory of weak interactions can be consistently transferred into our scheme. Finally, the possible existence of extremely weak interactions is speculated (metastability of matter and charge nonconservation)."

92. S. Sawada and M. Yonezawa

Progr. Theoret. Phys. (Kyoto) 22, 610 (1959) (received June 22, 1959) On the Possible Role of $\pi^{0}$-Meson in Decay Process

They analyzed the electron energy spectrum of $\mathrm{Ke}_{3}$ assumuing "I $\mathrm{O}^{\prime}$ rias $\approx 350$ Mes.

93. M. Sugawara and A. Kanazawa

Phys. Rev.115, 1310 (1959) (reccived July 2, 1959)

Meson-Meson Scattering Term in Pseudoscalar-Pseudoscalar Meson Theory

94. V. S. Barashenkov

Zhur. Eksptl。i Teoret. Fiz. 3\%, 1464 (1939); Suviel Phys. JETP 10, 1038 (1959) (received July 13, 1959)

Angular Asymmetry in $\pi, N$ Collisions and $\pi, \pi$ Interaction

95. I. Sato, A: Takahashi and Y. Ueda

Progr. Theoret. Phys。(Kyoto) 22, 617 (1959) (received July.20, 1959)

Properties of the Pion-Pion Interaction Derived from the Analysis of Pion-Nucleon Scatterings

"... we are led to the conclusion that in the isotopic spin state $I=0$ of the pion-pion system, the pion-pion interaction is attractive and its strength is such that the scattering length is of the same. order of magnitude as the pion Compton wave length, and that in the isotopic spin state $I=1$, the interaction is also attractive and is of strength at least comparable to that of the pion-nucleon interaction in the $(3,3)$ state." 
96. A。D. Galanin

Zhur. Eksptl. i Teoret. Fiz. 38, 243 (1960); Soviet Phys. JETP. 11 ;

$177,(196,0)$ (received July 22, 1959)

On Meson-Nucleon Scattering in Large Orbital Angular. Momentum States

" $\%$ if the as sumption is made that the $\pi \pi$ scattering amplitude exhibits no resonance at low energies, then a disagreement with experiment is obtained.!!

97. M. Ikeda, S. Ogawa, and.Y. Ohnuki

Progr. Theoret. Phys. (Kyoto) 22, 715 (1959) (received July 28, 1959)

A Possible Symmetry in Sakata's Model for Bosons-Baryons System

: ". From our theory the following are obtained:

$\therefore\left(\right.$ a) iso-singlet $\pi_{0}^{l}$ - meson state, which is a pseudo-scalar, exists,

(b) the spin of $\Xi$ particle may be $(3 / 2)^{+}$, and

(c) several resonating states in $\mathrm{K}$ - and $\pi$-nucleon scattering are anticipated to exist.

98. V. S. Barashenkov

Nuclear Phys。15, 486 (19.60) (received Aug。4, 1959)

Nonelastic Collisions of Fast $\pi$ Mesons with Nucleons and Peripheral $\pi \pi$ Interactions

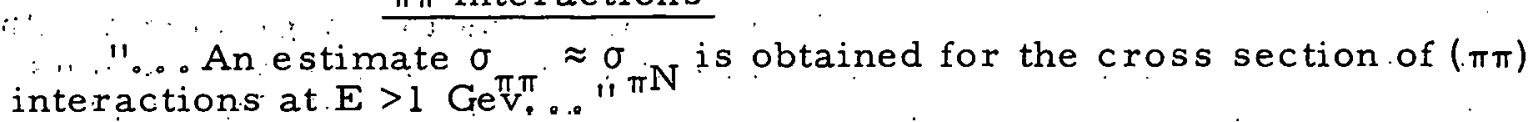

99. F. Cerulus

Nuovo cimento 14, 827 (1959) (received Aug. 21, 1959)

Consequences of a Strong $\pi-\pi$. Interaction in the Statistical Theory of p-p Annihilation

"The Fermi statistical model for multiple particle production is applied to the p-pannihilation process; calculations are performed for three assumptions on the $\pi-\pi$ interaction, viz,; (a) a strong resonance in the state $1=1, T=1$ with total energy $4 \mathrm{~m}_{\pi} ;(\mathrm{b})$ the same with total energy $3 \mathrm{M}_{\pi}$;

(c) no interactions. Multiplicities and spectra for 2, 4, and 6-prong $\pi^{\prime}$ tars are computed. The $\pi-\pi$ isobar model (a) z̧grees well with experiment, taking an interaction volume

$$
\dot{\Omega}_{0}=\left(\frac{\hbar}{m_{\pi} c}\right)^{3} \frac{4 \pi}{3} \text {. }
$$

The two-prong spectrum shows a hump around $7,60 \mathrm{Mev}$ kinetic energy."

100. V."I。 Rus'kin and P.A. Usik

Zhur。Eksptl。i Teoret. Fiz. 38, 929 (1960); Soviet Phys。JETP 11 , 669 (1960) (received Sept. 18, 1959)

The Significance of'Strange Particles in Fermi's Statistical Theory

"It is shown that account of resonance interaction of two $\pi$ mesons in Fermi's statistical model permits one to explain a number of experimental facts..." 
101. J. Wess

Nuovo cimento 15, 52 (1960). (received Sept. 21,1959 ).

Investigation of the Invariance Group in the Three Fundamental Fields Model

"For a theory in which elementary particles are represented by products of fundamental fields, consequences of an invariance group are investigated. The invariance group is the th ree-by-three unitary group. Tensor-calculus is used: to investigate the representations. Quantum numbers are defined and it is tried to identify certain families of elementary particles with irreducible representations."

102. K. Igi

Progr. Theoret. Phys. (Kyoto) 23, 170 (1960) (received Sept. 23, 1959) Possibility for or Against the Existence of a Neutral Scalar Meson

"... The neutral scalar meson, even if it existed at all, would play only a minor role in the world of strong interactions."

103. R. Hagedorn

Nuovo cimento 15, 246 (1960) (received Sept. 26, 1959)

Particle Production in 6.2-Gev p-p Collisions Treated by a Statistical

$\therefore$.

"...the result (inclusion of $\pi-\pi$ interaction) leads to disagreement with experiments. : It is hard to find a reason which rules out $\pi-\pi$ interactions in nucleon-nucleon collisions, but makes it important in annihilations."

104. S. Okubo

Phys. Rev. 1i , 357 (1960) (received. Sept. 28, 1959)

Pion-Pion and Pion-Kaon Scattering

105. Y. Fujii and S. Furuichi

Progr. Theoret. Phys. (Kyoto) 23, 251 (1960) (received Oct. 2, 1959).

Contributions from the Three-Pion State to the Axial Vector Coupling Constants in $\beta$ Decay

"... The three-pion state is considered as the simplest state which might improve the result of the static theory which gives GA/GV $<1 . . "$

106. W. Frazer and J. Fulco

Phys. Rev. 117, 1603 (1960) (received Oct. 9, 1959)

Partial - Wave Dispersion Relations for the Process $\pi+\pi \rightarrow \mathrm{N}+\overline{\mathrm{N}}$

"The problems of pion-nucleon and nucleon-nucleon scattering and nucleon electromagnetic structure involve the matrix element for two pions producing a nucleon-antinucleon pair. By use of the Mandelstam representation, we were able to write dispersion relations for the partial wave.

scattering amplitudes of thi's process."."

107. W. Frazer and J. R. Fulco

Phys. Rev. 117, 1609 (1960) (received Oct. 9, 1959)

Effect of a Pion-Pion Scattering Resonance on Nucleon Structure. II. (See also Ref. 86.) 
"It is shown that a resonance of suitable position and width in the $J=1$, $I=1$ state of the pion-pion system can bring the dispersion theoretic calculation of the isotopic-vector part of the nucleon electromagnetic structure into agreement with experiment.. The calculation of the isotopicvector part of the nucleon form factors involves in first approximation the pion form factor and the matrix element for the production by two pions of a nucleon-antinucleon pair. For the pion form factor we used a semi phenomerological solution based on the work of Chew and Mandelstam and involving two parameters related to the position and width of the resonance. For the $\pi+\pi \rightarrow: N+\bar{N}$ amplitude we used the results of the preceding paper."

108. J. Hamilton and W:S. Woolcock

Phys. Rer。 118, 291 (1960) (received Oct。22, 1959)

Low-Energy Pion Phenomena

$\because \because$ The possibility of detecting a $\pi=\pi$ interaction by low-energy pion scattering is examined."

109: V.M. Maksimenko

Zhur. Eksptl。i Teoret. Fiz。38, 652; Soviet Phys。JETP 11, 469 (1960) (received Nov。16, 1959)

On the Question of a Resonant $\pi-\pi$ Interaction

"... the angular correlation of $\pi$ mesons is analyzed for the case of stopped antinucleons... $\bar{p}+p \rightarrow \pi^{+}+\pi^{-}+\pi^{0}, \bar{N}+N \rightarrow 3 \pi$, and $\bar{N}+N \rightarrow \sigma+\pi \rightarrow$ $3 \pi$ 。

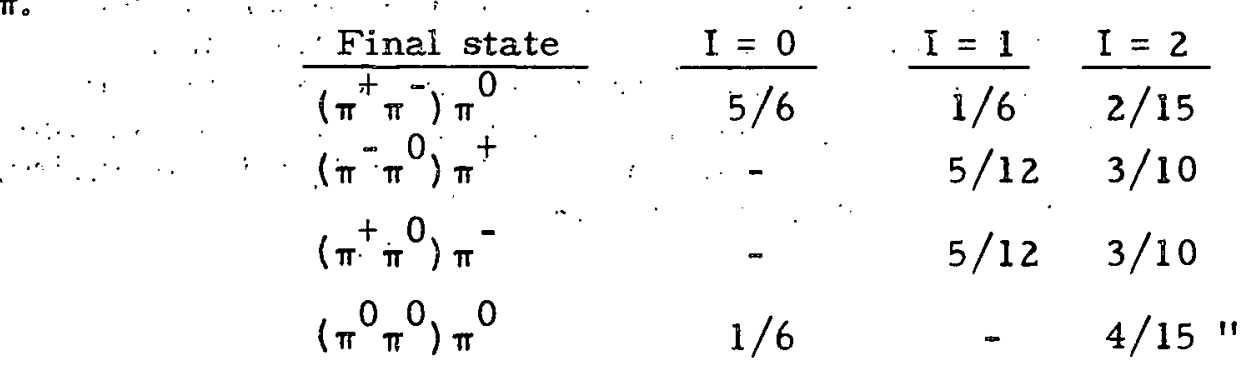

110. Bonsignori and Selleri

Nuovo cimento 15, 465 (1960) (received Nov。24, 1959)

Pion Cloud Effects in Pion Production Experiments

"A simple model is proposed for the study of single pion production in collisions of a generic particle. "a" on a target nucleon. The model consists essentially in neglecting all interactions but the one between the incoming particle and a single pion of the nucleon cloud. Application to a recent pion production experiment in proton-proton collision by the Birmingham group gives support to the plausibility of the idea. Another application to the : experiment by: the Bologna group gives qualitative evidence of pionpion interaction." Experimental data suggest a pion-pion cross section of the order of $10 \mathrm{mb}$. 
111. M. L. Goldberger and R. Oehme Ann. Phys. 10, 153 (1960) (received Dec. 7, 1959)

Application Dispersion Relations to Nucleon-Nucleon Scattering: The Two-Pion Contribution

112. M, Baker and F. Zachariasen

Phys. Rev. 118, 1659 (1960) (received Dec. 21, 1959)

Pion-Pion Scattering in the $\phi^{4}$ Theory

"Pion-pion scattering has been calculated using the determinantal method, assuming that a relativistic $(\lambda / 4)\left(y_{i} y_{4}\right)^{2}$ coupling is responsible for the interaction... The best fit of $\lambda$ obtained by this fit is unfortunately so large that the validity of the determinantal method is doubtful."

1.13. T. Sakuma

Progr. Theoret. Phys。(Kyoto) 23, 810 (1960) (received Dec.25, 1959) Pion-Pion Interaction and Pion Production in Pion-Nucleon Collision

"The evidence for the $\pi-\pi$ inleraction is investigated by considering the angular distribution of nucleons in pion production by pion-nucleon collision."

\section{G。 F. Chew}

Phys. Rev。 Letters 4, 142 (1960) (received Dec。28, 1959)

Three-Pion Resonance or Bound State

"The purpose of this note is to point out that it is not unreasonable to expect a three-pion resonance or. even a bound state at roughly the same energy as the two-pion resonance. The essential point is that in the particular three-pion state involved in electromagnetic structure, each pair of pions feels the same strong attractive force as that producing the two-pion resonance. The three-pion state has $\mathrm{I}=0, \mathrm{~J}=1$ and odd parity... Such a vector meson has been discussed by INàmbu. $\dagger$

${ }^{*}$ Ref.70, Ref. 73 .

$\dagger_{\text {Ref. } 68 .}$

115. A. Io Lebedev and V。 A。 Petrun'kin

Soviet Phys. JETP 11, 962 (1960) (received Jan。 3, 1960)

Formation of Pions in $\pi-\mathrm{N}$ Collisions

"... it is possible to describe the experimental data just as well without taking into account the meson-meson interaction, but including instead in the statistical theory the effect of the finite width of the isobar which decays to give $\pi$ mesons."

116. K. Ishida, A. Takahashi, and.Y. Ueda

Progr. Theoret. Phys. (Kyoto).23, 731.(1961) (received Jan.6, 1960) Pion-Pion Interaction and.Pion- $\overline{N u} c l e o n$ Scattering

"In the isotopic spin state I = 0 (S wave) of pion-pion system pion-pion interaction is attractive and the scattering lenght is of the order of one pion Compton wave length, while in the $I=1$ ( $P$ wave) of pion-pion state a definte conclusion could not be obt ained." 
117. M. L. Good and W. G. Holladay

Phys. Rev. Letter.s 4, 138 (1960) (received Jan. 11,'1960)

$T=1 / 2$ Selection Rule and $\mathrm{K}_{2 \pi}+$ Decay

$\therefore$ i... A discrepancy of . . 100 in the rates exists if the $T=1 / 2$ rule is taken to be exact. The point we, wish to make here is that there is a natural resolution of this discrepancy in the recently proposed strong attraction between two pions in the $\mathrm{T}=2, \mathrm{~J}=.0$ state at low energy."

118. G. F. Chew and S. Mandel stam

Phys. Rev. 119, 467 (1960) (received Jan. 18, 1960)

- Theory of the Low-Energy Pion-Pion Interaction

"The double dispersion repr. is applied to the problem of $\pi-\pi$ scattering, "and it' is shown that, if inelastic effects are important only at very high energies and S-wave scattering dominates at low energy, a set of integral. equations for the low-energy amplitudes can be derived..."

119. G. Chew, S. Mandelstam, and H. P. Noyes

Phys. Rev. 119, 478 (1960) (received Jan. 18, 1960)

S-Wave Dominant Solutions of the Pion-Pion Integral Equations

"The integral equations for pion-pion scattering formulated by Chew and Mandelstam are put into a form suitable for numerical solution."

120. I. M: Dremin

Suviet Phys. JETP 12, 94 (1961) (received Feb. 1960)

Peripheral Interactions Between $\pi$ Mesons and Nucleons at High Energies

121. M. Cini and S. Fubini

Ann. Phys. 10; 352 (1960) (received Feb. 1, 1960)

Theory of Low-Energy Scattering in Field Theory

"... it is probable that a resonance in the $\mathrm{T}=1, \mathrm{~J}=1$ state is indeed compatible with the equations. We believe, however, that the two parameters of such a resonance are not determined by the equations. Whether such a resonance does exist cannot therefore be decided by studying the pion-pion problem alone, but rathe $r$ by investigating the effect of such a resonance in all other phenomena involving pions as virtual states."

122. A. A. Ansel'm

Soviet Phys. JETP 11, 1356 (1960) (received Feb.2, 1960)

Solution of Equation for the Meson-Meson Scattering Amplitude in the Asymptotic Region

123. V. A. Kolkunov, L. B. Okun', A。 P. Rudik, and V. V. Sudakov

Soviet Phys. JETP 12, 242 (1961) (received Feb. 6, 1960) (Presented

'. ' ' by Rudik'at the 1960 Rochester Conference on High-Energy Physics)

Nearest Singular: Points in the Pion-Pion Scattering Problem

124. J: Sakurai

Annals of Physics 11:: i (1960) (received Feb. 26, 1960)

Theory of Strong Interactions 
"It is assuming that our theory satisfied simultaneously almost all the principles that have been proposed on simple theoretical grounds by various deep thinkcrs of elementary particle physics. The theory is, in a certain sense, founded on Heisenberg's conviction that besides the selection rules and the invariance principles the only other guiding principle should be simplicity. It explains the profound idea anticipated by Schwinger that internal attributes such as baryonic charge ( = his nucleonic charge) should have 'dynamical manifestations.' It fulfills the dream of Wigner and Gell-Mann that the re ought to exist a universal coupling related to baryon conservation. It answers Pauli's question in January 1957 'Why does the Lord still appear to be right-left symmetric when he expresses himself strongly?' and at the same time satisfies Lee and Yang who argue that a satisfactory answer to Pauli's question should not depend on the detailed structure of the interaction Lagrangian. It realizes both the Pais principle of economy of constants and the Pais principle of a hierarchy of interactions in a natural and elegant manner, and it somehow reminds us of Feynman's remark that one should generate new ideas by asking what would happen if history were different."

125. J. Bowcock, N. Cottingham, and D. Lurie

Nuovo cimento 16, 918 (1960) (received March 15, 1960)

Effect of a Pion-Pion Scattering Resonance on Low-Energy PionNucleon Scattering

"By suitable choice of pion-pion resonance parameters we were able to fit both the experimental pion-nucleon phase shifts and the nucleon electromagnetic form factors."

126. N. N. Khuri and S. B. Treiman

Phys. Rev. 119, 1115 (1960) (received March 16, 1960)

Pion-Pion Scattering and $\mathrm{K}^{+} \rightarrow 3 \pi$ Decays

". $. a_{2}-a_{0} \approx 0.7$ in units of the pion Compton wave length."

127. D. Amati, E. Leader, and B. Vitale

Nuovo cimento 17, 68 (1960) (received March 21, 1960)

Theory of Low-Energy Nucleon-Nucleon Scattering. I.

"... The spectral functions are calculated using unitarity both in the nucleon-nucleon and in the nucleon-antinucleon channels. In the latter the two-pion contribution is calculated in terms of pion-nucleon scattering and contains amplitudes characterizing the low waves of the $N \bar{N} \rightarrow 2 \pi$ process."

\section{J.J. Sakurai}

Nuovo cimento $\underline{16}, 388$ (1960) (received March 24, 1960) Pion Resonances

"The three pions that are capable of producing anything like the hard core effect must necessarily be correlated. Using an elementary argument one can convince oneself that, if the repulsive core (atiractive well) in $\mathrm{NN}(\mathrm{N} \overline{\mathrm{N}}$ ) interactions is to exist in all angular momentum and parity states it must be due to a state which has the same symmetry properties asia

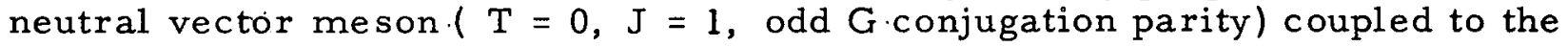


nucleon via an effective vector-type coupling. We can readily construct a $\pi^{+} \pi^{-} \pi$ (but not a $3 \pi^{0}$ system) with the desired symmetry properties. In this manner we are led to the view that there exists a three-pion resonance in $\mathrm{T}=0, \mathrm{~J}=1.1$ i

129. E. V.. Teodorovich

Soviet Phys. JETP 12, 334 (1.961) (received March 24, 1960)

Pion-Pion Interaction and the Electromagnetic Structure of the Nucleon

130. F.' Selleri

Nuovo cimento 16, 775 (196.0) (received March 30, 1960)

Evidence for Two Pion-Pion Resonances

"... In this note we want to point out that a careful analysis of the

existing experimental information" on high energy $\pi^{ \pm}-p$ scattering suggests

the existence of two pion-pion resonances in the isotopic spin states $I=1$ and $I=2$. First of all we will disciuss the 0.9-Gev maximum in $\sigma^{-}$. We will see that the only explanation of it which does not contradict any

: I: existing experimental fact is in terms of a strong pion-pion interaction in the $: I$ = 1 state, in full agreement with current theoretical ideas (Chew and Màndelstam,. Ref, 118; Frazer and Fulco, Refs, 86, 107). Noting, furthermore, the striking similarity between this maximum and the one in $\sigma^{-}$at 1. $3 \mathrm{Gev}$, we will deduce the $I=2 \pi \cdot \pi$ resonance should exist."

131.K. Itabashi, M. Kato, K. Nakagawa, and Go Takeda Progr."Theoret. Phys: (Kyoto) 24, 529 (1960) (received April 5, 1960) $\because: \quad$ (also given at 1960 Rochester Conference) The Theory of the Nucleon Level Structure in Terms of the $\pi-\pi$ Resonance

$\therefore$ : "The theory is based on two strong interactions, the usual static pionnucleon interaction and the pion-pion resonant interaction in $T=J=i$ state. "

132. W. Frazer and J.Fulco

Phys. Rev. 119;1420 (1960) (.received April 8, 1960)

Partial - Wave Dispersion Relations for Pion-Nucleon.Scattering

133. R。 F. Sawyer and K. C. Wali

Phys. Rev. 119, 1429 (1960).(received April 11, 1960)

Pion:-Pion Interactions in $\tau$ and $\tau^{\prime}$ Decays

134. $P$. Carruthers and $H . A$. Bethe :

Phys. Rev. Letters 4, 536 (1960) (received April 18, 1960)

Role of the $\pi-\pi$ Interaction in High-Energy $\pi$-Nucleon Interactions

"The ratio of the $t=1 \pi-\pi$ amplitude to the $t=0 \pi-\pi$ amplitude, as deduced from $r=\sigma(n+-) / \sigma(p-0)$, goes through a distinct maximum near. $900 \mathrm{Mev}$. We consider this to be evidence for the $t=1 \pi-\pi$ resonance suggested by Frazer and Fulco." :.,

\section{J. J. Sakuiraì}

Phys. Rev. 119, 1784 (1960) (received April 18, 1960)

Spin-Orbit Force and a Neutral Vector Meson 
"The coefficient of $\left(\underline{\sigma}_{1}+\underline{\sigma}_{2}\right)$. $\hat{n}$ in the pp scattering amplitude at 310 Mev can be completely accounted for by a strongly interacting neutral vector meson (or a sharp three-pion resconance in the $T=0, J=1$ state) of mass $\approx 3 \mu \pi$ to $4 \mu \pi . "$

136.K. Hiida and N, Nakanishi

Progr. Theoret Phys. (Kyoto) 24, 414 (1960) (received April 23, 1960)

Electromagnetic Structure of the Nucleon. V.

"Numerical results of the $3 \pi$-state contributions to the isoscalar part of the electromagnetic structure of the nucleon."

137. B. W. Lee and M. T. Vaughn

Phys. Revo Letters. 4, 578 (1960) (received April 25, 1960)

$\underline{P}$-Wave Resonance in Pion-Pion Scattering

"... If one assumes the existence of a vector boson of isotopic spin one, which interacts with the isotopic vectur part of the pion current in the Lagrangian, and inslsts thal. the relluimalized mas of the postulated partiche be larger than twice the pion mass, then the particle will become unstable and one has not changed the selection rules of the theory from those of the conventional pseudoscalar meson theary. Such a theory, on the other hand, will necessarily predict a $p$ wave resonance in pion-pion scattering: "

138. V. I. Gol'danskii and V. M. Maksimenko

Soviet Phys. JETP 12, 584 (1961) (received April 26, 1960)

The Hypothesis of the Neutral $p$ Meson in Light of Data on ProtonAntiproton Annihilation

"The data presently available on the yield of $\pi^{+}, \pi^{-}, 0^{2}$ and $\pi^{0}$ me sons during annihilations are inconsistent with the existence of a $\pi_{0} 0_{(\rightarrow 2}()_{)}$meson with a mass smaller than $3.5 \mathrm{~m}$ and also with the existence of a $\left.\pi^{0} 10^{(\rightarrow 3 \gamma}\right)$ meson with a mass smaller than $5.5 \mathrm{~m}_{\pi^{\circ}}$. On the other hand $\rho \rightarrow \pi^{+}+\pi^{-}+\gamma$ does not contradict the experimental data.

139. M. Sugawara and A. Kanazawa

Phys.Rcv。119, 2074, (1960) (received May 4, 1960)

Meson-Meson Scattering Term and.Low-Energy Pion-Nucleon Scattering

140. A. N. Mitra

Nuclear Phys. 18, 502 (1960) (received May 16, 1960)

Pion-Pion Effects in $\tau$ Decay

"... An $I=0$ interaction is by no means ruled out by experiment."

141. G。 Breit

Phys. Rev. 120, 287 (1960) (received May 27, 1960)

Nucleon-Nucleon Spin-Orbit Interaction and the Repulsive Core

"Recent proposals to explain the phenomenological repulsive core and spin-orbit interaction in nucleon-nucleon scattering in terms of a vector meson field are discussed... A fit to the Bryan potential indicated a mass of the vector. meson between $9 \mathrm{~m}_{\pi}$ and $12 \mathrm{~m} \pi^{\circ} "$ Breit refers to the $W$ as a "heavy photon:" (See also G. Breit, Proc."Natl. Acad. Sci. 46, 746 (1960)) 
142. S. C. Erautschi and J. D. Walecka Phys. Rev. 120, 1486 (1960) (received June 6, 1960) Pion-Nucleon Scattering in the Mandelstam Representation

143. F. Salzman and $G_{0}$ Salzman

Phys. Rev. 120, 599 (1960) (received June 6, 1960)

Pion Production from $\pi^{-}+p$ Collisions in the Long-Range Interaction Model

"... this pion exchange leads, in single pion production, to an "excited state" $\pi$ which decays into two pions..."

144. Y. Batúsov, S. Bunyatov, V. Sidorov, and V. Yarba Soviet Phys. JETP 12, 354 (1961) (received June, 8, 1960) Determination of the -Charge Exchange Cross Section: for Pion-Pion Collision from the Analysis of the $\pi+p \rightarrow \pi+\pi^{+}+n$ Reaction at $290 \mathrm{Mev}$

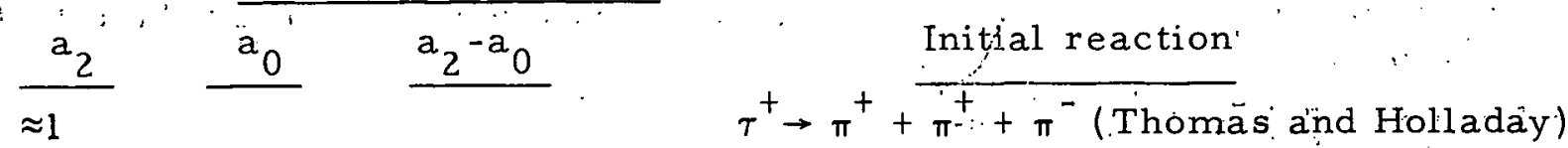

$$
\begin{aligned}
& -(0.35 \pm .30) \quad \pi^{-}+p \rightarrow \pi^{-}+\pi^{+}+n \text { (This work) } \\
& \begin{array}{llll}
-0.48 & -0.8 & 0.3 & K^{ \pm} \rightarrow 3 \pi \text { (Sawyer and Wali) }
\end{array} \\
& -0.3 \ldots-1,0.7 \quad \mathrm{~K}^{ \pm} \rightarrow 3 \pi \text { (Kuhri and Treimar) } \\
& 1 \pi+N_{i} \rightarrow \pi+N(\text { Efremov, Mescheryokov, } \\
& \text { and Shurkov, JETP) } \\
& 1 \quad \pi+N \rightarrow \pi+N \text { (Ishida, Takahashi, } \\
& \text { and Ueda) }
\end{aligned}
$$

145. S. Kamefuchi

- Nuclear Phys. 18,691 (1960) (received June 7, 1960) On Salam's Equivalence Theorem in Vector Meson Theory

"Some comments are... made on renormalizability of vector meson fieldṣ.".".

146. V. S. Barashenkov

Nuclear Phys。22, 71 (1961) (received June 10, 1960)

Inelastic Interactions of $\pi$ Mesons with Nucleons at $6.8 \mathrm{Gev}$

". . within the accuracy of present-day theory and experiment there is no. need to take the resonant $\pi-\pi$ interaction into account in the analysis of $(\pi, N)$ and $(N, N)$ collisions."

147. Y. Miyamoto

Progr. Theoret. Phys. (Kyoto) 24, 840 (1960)(received June 11, 1960) $\therefore$ P-Wave Pion-Pion Interaction 
"P-wave pion-pion resonance formula is derived by both the ChewMandelstam theory and the chain approximation."

148. A. N. Mitra, R. P. Saxena, and P. Narayanasiwamy Nuclear Phys. 20, 491 (1960) (received June 16, 1960)

A Note on $\pi-\pi$ Interaction

"The numerical solutions of the $\pi-\pi$ equations... agree qualitatively with the predictions and suggest that the $\mathrm{I}=0$ interaction (which has the larger coupling parameter) gives a resonance at a much higher energy than $\mathrm{I}=2$."

149. D. Amati, E. Leader, and B. Vitale Nuovo cimento 18, 409 (1960) (received July 20, 1960)

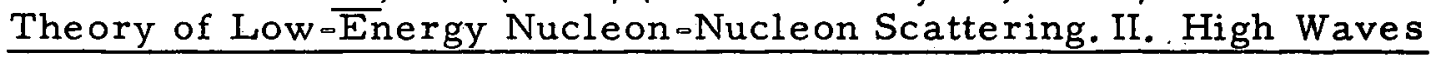

"The se formulae contain explicitly. the effect of the Born term and rescattering corrections in pion-nucleon scattering as well as the effect of a possible pion-pion interaction."

150. P. A. Usik and V. I. Rus'kin

Soviet Physics JETP 12, 1200 (1961) (received June 21, 1960)

Peripheral $\pi-\pi$ Interactions Between $\cdot$ High-Energy Nucleons

151. J. S. Ball

Phys. Rev. Letters 5, 73 (1960) (received June 27, 1960)

Effect of the Pion-Pion Resonance on the Negative-Positive Ratio

152. Ding-chang Hsien, Tso-hsiu Ho, and W. Zöllner

Soviet Phys. JETP 12, 1165 (1961) (received June 31, 1960)

Low-Energy Integral Equations for $\pi-\pi$ Scattering

"A critical analysis of the Chew-Mandelstam equations for $\pi-\pi$ scattering is presented."

153. A. V. Efremov, V. A. Meshcheryakov, D. V. Shirkov, and Hun-yuan Tsu

Nuclear Phys: 22, 202 (196 ) (received July 1, 1960) (presented by

D. V. Shirkov, 1960 Rochester Conference).

The Derivations of the $\pi-\pi$ and $\pi-N$ Scattering from the Mandelstam Representation

$\begin{array}{llll} & \text { Expt. } & \text { Theory }: & \frac{2 \mathrm{M} \psi_{0}=0.12}{\mathrm{a}_{31}} \\ \mathrm{a}_{13} & -0.032 \pm .002 & -0.14+2 \mathrm{M} \psi_{0} & -.02 \\ \mathrm{a}_{11} & -0.044 \pm .005 & -0.16+2 \mathrm{M} \psi_{0}-\psi_{1}-.04-\psi_{1} \\ & -0.038 \pm .038 & +0.075-2 \mathrm{M} \psi_{0}+2 \psi_{1}-.045+2 \psi_{1}\end{array}$

By inspection of the above table, one can see that the large experimental errors make it impossible to draw any conclusion about the integral $\psi$, 
which depends on the $p$-wave of $\dot{\pi}-\pi$ scattering. However, the sign and order of magnitude of $\psi_{0}$ can be considered to be reliable. This result is in accordance with the conclusions of Ishida, Takahaski, and Ueda.

154. D. Amati, E. Leader, and B. Vitale

Nuovo cimento 18,458 (1960) (received July 7, 1960)

Theory of Low-Energy Nucleon-Nucleon Scattering. III. Partial -Wave Integral Equations for Low-Orbital-Momentum Amplitudes

"... Integral equations for the se amplitudes are constructed, and the entire two-meson contribution to the left-hand cut is explicitly given in terms of the effect of the Born term and 3,3 resonance in pion-nucleon scattering and a possible $\pi-\dot{\pi}$ interaction."

155. J. Hamilton and T. D. Spearman

Ann. Phys。12, $172(1961)$ (received July 8, 1960)

Dispersion Relation for the s-Wave Partial Amplitude in Pion-Nucleon Scattering

"... The energy dependence of the discrepancy implies that there is an appreciable contribution from the process $\pi+t \rightarrow \mathrm{N}+\overline{\mathrm{N}}$. Comparing the values in the $T=1 / 2$ and $T=3 / 2$ isotopic spin scattering states, it is clear that the process $\pi+\pi \rightarrow N+N$ must contribute in the $T=0$ isotopic state: The energy dependence of the $(\pi+\pi \rightarrow N+N)$ contribution suggests that the large values of the $\pi-\pi$ scattering amplitude occur at an energy no greater than about $12 \mu^{2}$."

156. Hung-yuan Chou

Zhur. Eksptl。i Teoret。Fiz. 40, 227 (1961); Soviet Phys. JETP 13, 156 (1961) (received July 9, 1960)

Intégral Equation for Pion-Pion Scattering at Low Energies

157. V: S. Barashenkov

Zhur. Eksptl。i Teoret。Fiz。40, 1313 (1961); Soviet Phys. JETP 13, 925 (1961) (received July 9, 1960)

Pion Interaction in the Fermi Statistical Theory

"On the assumption of a resonance $\pi-\pi$ interaction the energy spectra are calculated for the $\pi$ mesons and nucleons produced in inelastic $N-N$ collisions at $\mathrm{E}=9$ Bev。"

158. Y. Simonov and K. A. Ter-martirosya

Soviet Phys. JETP 12, 1003 (1961) (received July 23, 1960)

Semi-Analytical Solution of Equations of the Chew-Mandelstam Type

159: S. C. Frautschi

Phys.' Rev。 Letters, 5, 1.59 (1960) (received July 26, 1960)

Sensitivity of Low-Energy Pion-Nucleon Scattering to Pion-Pion

Resonance

$\because \quad \because 47$ 
160. S. Ishida

Progr. Theoret. Phys. (Kyoto) 24, 1262 (received July 27, 1960)

Pion-Nucleon Scattering and Pion-Pion Interaction

161. J. D. Soloviev

Zhur. Eksptl。i Teoret. Fiz。 40,597 (1961); Soviet Phys. JETP 13, 418 (1961) (received $\overline{\text { Aug. }}$ 13, 1960)

Photoproduction of Pions on Pions

"... It has a.resonant character if resonance occurs in the scattering of pions on pions in a state $\mathrm{J}=\mathrm{I}=1 . "$

162. B. De Tollis, E。 Ferrari, and $H_{\text {. }}$ Muczek

Nuovo cimento 18,198 (1960) (received Aug. 16, 1960)

The Influence of Possible Pion-Pion Interaction on the Photoproduction of Charged Pions

"In this paper we will show that the theoretical predictions on the $\pi^{-} / \pi^{+}$ ratio at $90^{\circ}$ can be brought into better agreement with experiment by taking into account the presence of a possible pion-pion interaction, which has already been investigated by several authors. This interaction is supposed to take place in a resonant $\mathrm{T}=1, \mathrm{~J}=1$ state."

163. M. Gourdin, D。 Lurie, and A. Martin

Nuovo cimento 18,933 (1960) (received Aug. 5, 1960)

Effect of a Pion- Pion Resonance on Low-Energy Meson Production

164. S. Sawada

Progr. Theoret. Phys。25, 83 (1960) (received Aug。20, 1960)

Single-Pion Production Process in Pion-Nucleon Collision and the Sakalia Mndel

"We calculate the energy and angular distribution of the pion and nucleon which will be useful for obtaining information about the $I=2$ boson isobar and $I=3 / 2$ fermion isobar."

165. A. Komar and A. Salam

Nuclear Phys。21, 624 (1960) (received Aug. 22, 1960)

Renormalization Problem for Vector Meson Theories

166. G. F. Chew

Proceedings of the 1960 Annual International Conference on High-Energy Physics at Rochester (Conference held Aug. 25 -Sept. 1) p. 273

The Pion-Pion Interaction

"...Qualitatively the force picture is as follows: (1) Exchange of I = 2 (s-wave) pairs can never be important because of the low statistical weight of this state. (2) Exchange of $I=2$ (s-wave) pairs might be important if scattering in this state were strong; it turns out, however, that the $I=2$ phase shift never becomes large. (3) That leaves the long-range force due to $I=1$ (p-wave) pair exchange, and this force is very strong if there is a 
p resonance. It is attractive for the $I=2$ state and repulsive for the $I=0$ state: for the $I=1$ state it changes sign, being repulsive at very long range and attractive in the intermediate and short-range region."

167. R. J。Eden

Proceedings of the 1960 Annual International Conference on High-Energy Physics at Rochester (Conference held Aug. 25-Sept. 1), p. 219 The Use of Perturbation Methods in Dispersion Theory

"... I will mention two experiments on which the complication of closed loop resonances may cause trouble. One is the pion-production experiment $\left.\pi^{-}+p \rightarrow n+\pi^{-}+\pi^{+}\right)$. . . The second experiment is on the reaction $\mathrm{p}+\mathrm{d} \rightarrow \mathrm{He}^{3}+$ neutral system."

168.J. W. Moffat

Proceedings of the 1960 Annual International Conference on High-Energy

Physics at Rochester (Conference held Aug。25-Sept。1), p。355 Resonance and the Unphysical Region

"On the basis of the Chew-Mandelstam partial-wave analysis of pion-pion scattering the partial -wave amplitude is determined in terms of the unitary condition and the branch cuts and poles of the inverse amplitude."

169. Y. Ohnuki

Proceedings of the 1960 Annual international Conference on High-Energy

Physics at Rochester (Conference held Aug. 25-Sept. 1), p. 84.3

Composite Model of Elementary Particles

"... In 1955, Sakata proposed a composite model for particles belonging to the baryon-meson family. According to it, three particles, proton, neutron and $\Lambda$ particle, are regarded as basic particles at the present stage, and others (belonging to this family) are assumed to be compound objects constructed out of them.

$$
M=3, M^{1}=0
$$

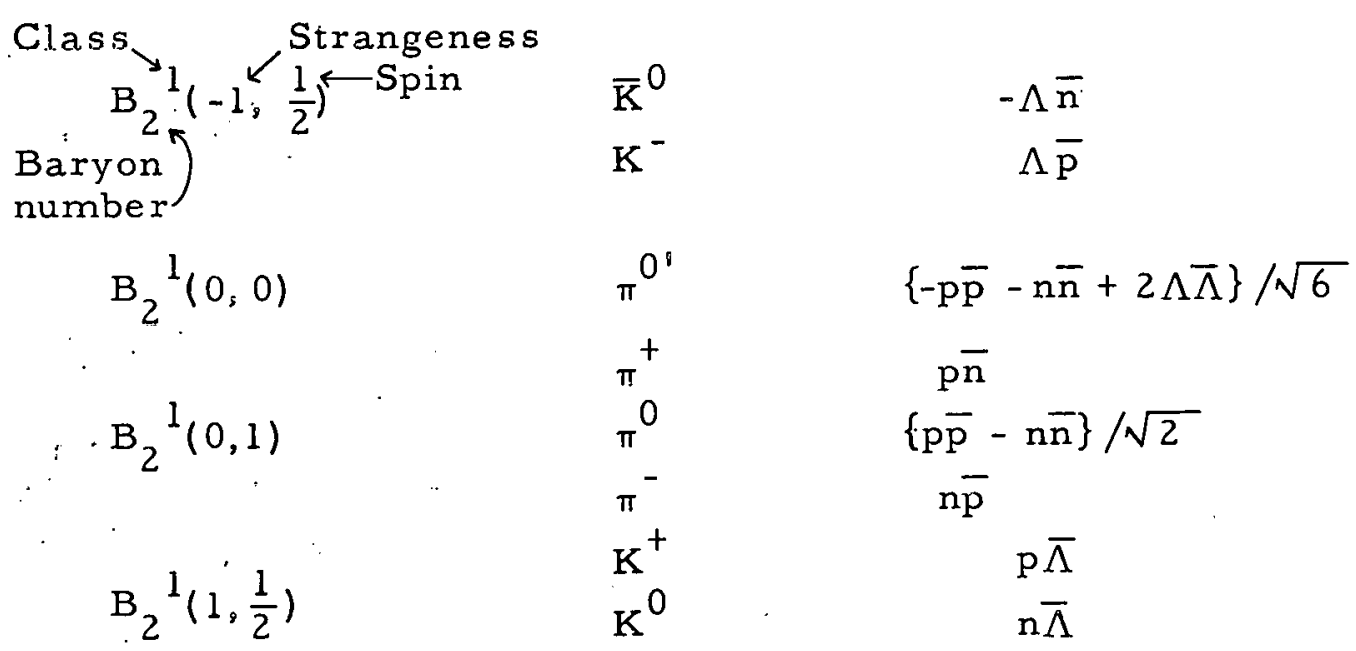


Other classes exist,

Class II: $\quad M=0, M^{p}=0$

$$
\mathrm{B}_{2}{ }^{2}(0,0) \frac{\mathrm{p} \overline{\mathrm{p}}+\mathrm{n} \overline{\mathrm{n}}+\Lambda \bar{\Lambda}}{\sqrt{3}} \text { particle has not been seen yet." }
$$

(It has now: this is the $\omega_{0}$ )

170. G. Pinski, E。C.G。 Sudarshan, and K。 T. Manhantappa

Proceedings of the 1960 Ann. International. Conference on High-Energy

Physics at Rochester. (Conference held Aug。25-Sept. 1), p. 173

Pion-Pion Interaction and Antinucleon Annihilalion

"A pion-pion resonance may be incorporated within the statistical

framework in a straightforward fashion by a covariant analogue of the

Breit-Wigner one level formula; we write for the differential transition

rate to an allowed configuration $\left\{p_{i}\right\}$ an expression which involves a resonance function of the effective mass of pairs of particles. The simplest such expression is

$$
1+\lambda^{2} \sum_{i>j} \delta\left[\left(p_{i}+p_{i}\right)^{2}-\mu^{2}\right]
$$

$\lambda^{2}$ and $\mu^{2}$ are parameters that have the dimensions of (mass) ${ }^{2}$."

171.G. Salzman and F. Salzman

Proceedings of the 1960 Annual International Conference on High-Energy Physics at Rochester (Conference held Aug。25-Sept. 1) p. 348

Single Virtual Boson Exchange Effects in High-Energy Collisions

172.J。W。 Moffat

Phys. Rev。121,926 (1961) (received Sept。2, 1960)

Determination of Pion Pion Scattering Amplitudes Satisfying Dispersion Relations and Unitarity

173. L。 Do Solov'ev, G。 Bialkowski, and A。 Jurewicz

Zhur。Eksptl。i Teoret。Fiz。 40,839 (1961) (received Sept。6, 1960)

Equations for Photoproduction of Pions on Nucleons with Effects Due To a Pion-Pion Interaction Taken into Account

174. J. Bowcock, No Cottingham, and D。 Lurie

Nuovo cimento 19, 142 (1961) (received Sept。 8, 1960)

The Effect of a Pion-Pion Interaction on Low-Energy Meson-Nucleon Scattering。TI。

"By confirming our considerations to the isotopic spin flip combination of waves we are able to isolate the effects due to a pion-pion interaction in the $T=1$ state. We find that such an interaction is definitely necessary to give agreement with experiment and that a simple resonance in the $\mathrm{J}=1$, $T=1$ state gives a good fit to the data." 
17.5.D. I. Blokhintsev and Wang Yung

Nuclear Phys. 22, 410 (1961) (received Sept. 9, 1960) $\pi-\pi$ Interactions in $\pi-N$ Collisions at High Pion Energies (about 7 Gev).

"In the case of inelastic collisions the contribution of the $\pi-\pi$ interaction is already quite noticeable and, therefore, it is possible to determine the cross sections for $\pi$ interactions.

$$
\begin{aligned}
& \sigma_{\pi \pi} \approx 15 \text { to } 30 \mathrm{mb} \text { at about } 680 \mathrm{Mev} / \mathrm{c} \text { in c.m. system of } \pi-\pi \\
& \left(\mathrm{M}_{\pi \pi}=2 \sqrt{(680)^{2}+(140)^{2}}=2 \times 695=1390 \mathrm{Mev}\right) . "
\end{aligned}
$$

176. J. Bowcock,W. N. Cottingham, and D. Lurie

Phys. Rev。 Letters 5, 386 (1960) (received Sept. 16, 1960) $\pi=\pi$ Seattering, Nucle on Structure, and $\pi-N$ Scattering

Use $t_{R}=22.4 \mu^{2}$. Spend most of time justifying why they are right and Frauts̈chi is wrong.

177. H. Wong

Phys. Rev. 121, 289 (1961) (received Sept. 28, 1960)

Effects of the Pion-Pion Resonance and the Three-Pion Resonance or Bound State on Neutral Pion Decay

178. Tso-hsiu Ho and Kuang-chao Chou

Soviet Phys. JETP 12, 1032 (1961)

On the Pion-Pion. Resonance in the P State

179

179. E。 Loman, S. Morris, E. Irwin, Jr。, and T. Truong

Ann. Phys. 13, :359 (1961) (received Oct. 5, 1960)

The Pion-Pion Interaction in $\dot{\tau}$. Decay

180. V. N. Strel'tsov

Zhur. Eksptl。i Teoret, Fiz。40, 1140 (1961); Soviet Phys. JETP 13, 802 (1961) (received Oct. 29, 1960)

Some Isotopic Relations for Reactions of the Type $\pi N \rightarrow \pi \pi N_{0}$

"Isotopic relations are utilized in an analysis of experimental data for reactions of the type $\pi N \rightarrow \pi \pi N$ from the point of view of resonant $\pi-\pi$ interactions。"

181. G. F. Chew and S. Mandelstam

Nuovo cimento 19,752 (1961) (received Oct. 31, 1960)

Theory of the LOW-Energy Pion-Pion Interaction. II.

"Self-consistent solutions can be found in which a $\mathrm{p}$-wave resonance is sustained by a "bootstrap" mechanism; that is, a strong attractive force in the $I=1$ state results from the exchange of a resonating pair of $p$-wave pions."

182. B.: W. Lee

Phys. Rev。12i, 1550 (1960) (received Oct. 31, 1960)

Two-Pion Exchange Mechanism in $\mathrm{K}^{ \pm} \mathrm{N}$ Scattering . 
$\mathrm{K}^{+}$"The exchange of two pions resonating in the $\mathrm{T} \cdot=1, \mathrm{~J}=. \mathrm{l}$ state between $\mathrm{K}^{+}$meson and nucleon in $\mathrm{K}^{+}-\mathrm{N}$ scattering is considered in double-dispersion representation."

183. Tubis and Üretsky

Phys。 Rev, Letters 5, 513 (1960) (received Oct. 31, 1960)

Interpretation of the Berkeley Anomaly in High-Energy $p=d$ Collisions

"It is our feeling that the $\therefore$ : data are consistent with the scatteringlength model ... and there is no necessity for invoking the existence of a resonance..."

184. J. Ball and D. Wong

Phys, Rev。 Letters 6, 29 (1961) (received Nov. 14, 1960)

$\mathrm{N} \overline{\mathrm{N}} \leftrightarrow \pi \pi$ Amplitude and the Electromagnetic Structure of the Nucleon

185.K. Ishida

Progr. Theoret. Phys。(Kyoto) 25, 294 (received Nov。 14, 1960).

Pion Interaction on the Pion-Nucleon Small p-Wave Phase Shifts.

186. Y. Fujii

Progr. Theoret. Phys。.(Kyoto) 25, 441 (1961) (received Nov, 22, 1960) Pion-Pion Interaction and Nuclear Forces

"Nuclear potentials arising from pion-pion resonance in the state $I=J \neq 1$ are calculated."

1.87. N. Kato

Progr. Theoret. Phys。(Kyoto) 25, 493 (1961) (received Nov. 28, 1960) Photopion Production and $(\gamma, 3 \pi)$ Interaction

188. J。 Uretsky and $\mathrm{T}$. Palfrey, $\mathrm{Jr}$.

Phys.Rev。121, 1798 (1961) (received Dec。14, 1960)

Photoproduction and Detection of Two-Meson Branch. State

189. M. Yamazaki

Progr. Theoret. Phys。 (Kyoto) 25, /2' (1461) (received Dec. 17, 1960) p-Wave Resonance in Pion-Pion Scattering

"By adjusting the coupling constants $22 / 4 \pi$ between pion and nucleon so as to fit the pion-pion scattering length $a=0.1 \approx 0.3 \mu^{-3}$ in the $I=1=1$ state we have found the resonance level of 600-400 Mev and resonance width of 140-40 Mev, which is quite consistent with (that) adopted by Takeda et al. in their theory of the second and third maxima in pion-nucleon scattering and also by Frazer-Fulco in the explanation of isotopic vector magnetic form factor."

190. P. Carruthers

Ann. Phys.14,229 (1961) (received Dec。22, 1960)

Pion Production and the Higher Resonances in Pion-Nucleon Scattering

"...For a $\pi-\pi$ resonance energy squared $S=10$, agreement withiexperiment is obtained with a width about one-third that suggested by nucleon electromagnetic structure." 
191.N.F。 Nelipa

Zhur。Eksptl。i Teoret.Fiz。40, 1085 (1961); Soviet Phys。 JETP 13,

766 (1961) (received $\overline{\mathrm{Jan}}$. 4, 1961)

Double Dispersion Relations and Photoproduction of Pions

192. G. Pinski

Phys。Rev。Letters 6, 136 (1961) (received Jan。9, 1961)

Test for Pion-Pion Resonance

"...As. n (the pion multiplicity) increases... [the relative contribution by the $\pi-\pi$ resonance diminishes] since there remains only one way for the pair (of pions) being correlated to be the resonant pair, while the total number of ways of choosing the resonant pair increases. Therefore, the effect of a pion-pion resonance is more prominent for $n$ small."

\section{3. $\mathrm{P}_{\mathrm{o}}$ : Carruthers}

iPhys。 Rev。122, 1949 (1961) (received Jan。11, 1961)

$\therefore$ Production of Pion Paires: Isospin Analysis

"... An experiment is suggested to measure the phase difference between even and odd $\pi-\pi$ isospin states, with a view toward detecting a resonance: in the $\pi-\pi$ system."

194. S. Sawada, To Ueda, and M。 Yonezawa

Progr. Theoret. Phys. (Kyoto) 25, 873 (1961) (received Jan。23, 1961) $\therefore$ The Single Pion Production. Process in $\pi-p$ Collision at 1 Bev and the

Sakata Model

"... It is shown that this generalized isobar model (Sakata model) can well explain the experimental momentum spectra. The evidence of boson isobars of $I=2$ with mass about $400 \mathrm{Mev}$ and $\mathrm{I}=1$ with mass about $650 \mathrm{Mev}$ are suggested from the analysis of the Derado-Schmutz data."

‘.195: M. Kawaguchi, M. Miyamoto, and Y. Fujii

Nuovo cimento 20, 408 (1961) (received Feb.2, 1961)

Effects of the $y \overline{-3} \pi$ Interaction on Photo Reactions

196. J. Hamilton, P。 Menotti, T。 D。Spearman, and W。S. Woolcock Nuovo cimento 20,519 (1961) (received Feb.6.'1961)

Evidence for Pion-Pion Interactions from $s$-Wave Pion-Nucleon Scattering

"By fitting the $s$-wave partial amplitudes for $\pi-N$ scattering, in an unphysical region, we have greatly extended the energy range over which we can examine the process $\pi+\pi \rightarrow N+\bar{N}$. Our method shows up contributions from this process in states of isotopic spin $T=0$ and $T=1$ 。

"The $\mathrm{T}=0$ amplitude is large but in the $\mathrm{T}=1 \mathrm{case}$ the amplitude is much smaller than the values which have been predicted from the nucleon isovector form factors."

197. S. Ohnuma

Progr。Theoret. Phys。(Kyoto) 25, 847 (1961). (received Feb。 8, 1961) Mass of Neutral Vector Meson and p-p Scattering 
198.J.G. Taylor

Phys. Rev。 Letters 6, 237 (1961)(received Feb. 10, 1961)

Pion-Pion Interaction and High-Energy $p+d$ Collisions

199. Y. Neeman.

Nuclear Phys,26, 222 (1961) (received Feb. 13, 1961) Derivation of Strong Interactions from a Gauge Invariance

"... This enables us to generate the strong interactions from a gaugeinvariance principle involving eight vector bosons."

200. R. G. Sachs and B. Sakita

Phys。Rev. Letters 6, 306 (1961) (received Feb.20, 1961)

Existence of the wo Particle

"We would like to point out that the existence of either a two-pion or a (bound) three-pion state of angular momentum $\mathrm{J}=0$ or 1 at this energy (approx $305 \mathrm{Mev}$ ) would appear to be inconsistent with observations on the $\mathrm{K}$ decay modes."

201. T. N. Truong

Phys.Rev。 Letters 6, 308 (1961) (received Feb.20, 1961)

Analysis of Anomaly in Double Meson Production in $\mathrm{p}+\mathrm{d}$ Collisions and the $s-$ Wave Pion-Pion Interaction

"The anomaly in double pion production observed by $A B C$ :may be explained by a nonresonant final-state interaction. .. in $\mathrm{T}=0$ (state) instead of assuming the existence of a neutral wo particle."

202.M. Bassetti

Nuovo cimento 20,803 (1961) (received Feb. 27, 1961)

Photoproduction of Neutral Vector Mesons

"We shall calculate here the cross section for the process $\gamma+p \rightarrow p+X$ where $X$ is a neutral vector meson."

203.J.G. Belifante

Phys.Rev.123, 306 (1961) (received Feb. 27, 1461)

Nucleon-Antinucleon Mechanism for Pion-Pion Resonances

204. B. W. Lee and $\mathrm{K}$. S。 Cho

Nuovo cimento 20,553 (1961) (received Feb.27, 1961)

Modified Static Equations for the $K_{\pi}$ Interaction Effect : of Pion-Pion

Resonance

205. I. Y. Kobzarev, L。 B. Okun, and I. Y. Pomeranchuk

Zhur。Eksptl。i Teoret, Fiz。41, 495 (1961) (received Feb。28, 1961)

(translation to appear in Soviet Phys. JETP 14, Feb. 1962)

Electromagnetic Interaction of a Neutral Vector Meson 
206. B. De Tollis and A. Verganelakis

Phys。Rev。Letters 6, 371.(1961) (received March 9, 1961)

Pion-Pion Interaction in the Photoproduction of Neutral Pions with

$$
\text { Polarized } \gamma \text { Rays }
$$

(Gives list of references.on $\pi-\pi$ interaction.)

"In this letter we shall see the effect of this interaction $(\mathrm{T}=\mathrm{l}, \mathrm{J}=1$, $\pi-\pi)$ in the case of $\pi^{0}$ photoproduction from the reaction $\gamma+p \rightarrow \pi^{\prime}+p$ with polarized $\gamma_{0} "$

207.S。 Bergia, A. Stanghellini, S. Fubini, and C. Villi

Phys. Rev. Letters 6, 367 (1961) (received March 13, 1961).

Electromagnetic Form Factors of the Nucleon and Pion-Pion Interaction

They predict a pion pion resunance with $\mathrm{T}=1, \mathrm{~J}=1, \mathrm{E}_{\mathrm{R}} \approx 4.7 \mathrm{~m}$. $\mathrm{T}=0, \mathrm{~J}=1$ particle could arise (Chew) from three $\pi^{i}$ s resonating two by two in the $T=0, J=1$ state. Its mass could be lower than $E_{R}$.

208:K. Kawarabayashi

Nuovo cimento 20,1030 (1961) (received March.13, 1961)

A Note on the Final-State Interaction in $\mathrm{K}_{2 \pi}^{+}$. Decay.

209. A. Salam and J。C. Ward

Nuovo cimento 20,419 (1961). (received March 13, 1961)

Vector Field Associated with the Unitary Theory of the Sakata Model

"... Thus in addition to the three original particles $(p, n$, and $\Lambda$ ), the theory would contain eight vector mesons: four. $\left(K_{\mu}\right)$ resembling $K$ mesons in their isotopic character, three $(\pi)$ resembling $\mu$ ions, and one particle $\left(\rho^{\prime}\right)$, an isoscalar. The $K$ could dectey into $K+r_{q}$. while $\rho$ might decay into $3 \pi_{0}$. The three-particle $\pi_{\mu}$ are identical with.the triplet ${ }^{\mu} \mathrm{B}_{\mathrm{T}}$ introduced
by Sakurai."

210.S. Sawada, T. Ueda, and M. Yonezawa

Progr. Theoret. Phys. (Kyoto) 25, 868 (1961)(received March 13, 1961) The $\tau^{+}$Decay and the Boson Isobar with $I=2$ of the Sakata Model

211. M. Gell-Mann

California Institute of Technology Synchrotron Laboratory Report CTSL-20, March 15, 1961)

The Eightfold.Way: A Theory of Strong-Interaction Symmetry

212. C. Goebel and $H_{0}$ J. Schnitzer

Phys. Rev。123,1021 (1961) (received March 16, 1961)

Pion Production and the Second Pion-Nucleon Resonance

"A model for the reaction $\pi+N \rightarrow 2 \pi+N$ at low energy which includes pion-pion interaction and final-state interactions in the 3,3 state is discussed. $\ldots$ The $\pi-\pi$. scattering lengths found are $a_{0}=-0.29 \mu^{-1}, a_{1}=0.122 \mu^{-}$, and $a_{2} / a_{0}=2 / 5$ by hypothesis." 
213. P. Budini and L。 Fonda

Phys. Rev。Letters 6, 419 (1961) (received March 20, 1961)

Pion-Pion Interaction from Threshold Anomalies in $\mathrm{K}^{+}$Decay

214. J. Wolf and W。 Zöllner

Dubna Report D-703, March 24, 1961

$\tau$ Decay and $\pi-\pi$ Interaction

215.B. H. Bransden and J。 W. Moffat

Phys. Revo Letters 6, 708 (1961) (received March 27, 1961)

Coupled s-and p-Wave Solutions for Pion-Pion Scattering

"We present here the results of numcrical iteration of the equations of Chew and Mandelstam when coupled s and $p$ waves are treated."

216. G. F。 Chew and S. C. Frautschi

Phys: Rev。123, 1478 (1961) (received March 30, 1961)

Dynamical Theory for Strong Interactions at Low Momentum Transfer But Arbitrary Energies

217. B. De sai

Phys. Rev. Letters 6, 497 (1961) (received March 31, 1961)

Low-Energy Pion-Pion s-Wave Phase Shifts

"It now becomes possible to make certain assertions about the $s$-wave $\pi \pi$ phase shifts on the basis of the crossing relations developed by Chew and Mandelstam."

218. M. Gell-Mann and F。Zachariasen

Phys. Rev。 124, 953 (1961) (received April 14, 1961)

Form Factors and Vector Mesons

"The $2 \pi$ and $3 \pi$ resonances are re-examined from the point of view that they are vector mesons coupled to conserved currents. The theory uf unstable mesons is discussed and formulas are then derived for the emission and propagation of these mesons. The connection with the electromagnetic form factors is then given, particularly for the simple case of infinite bare mass. The results are very similar to those of the dispersion method. Experimental manifestations of universality (connected with conserved vector current) are discussed. Applications are then made to the decay of $\pi^{0}$ and a group of related phenomena, including several "pole" experiments. Also the contribution of the $2 \pi$ resonance to $\pi-N$ scattering is discussed briefly from the vector meson point of view. Finally, we compare the vector meson approach to the alternative method using dispersion relations applied to presumably dynamical resonances."

219. P. Carruthers

Phys.Rev。Letters 6, 567 (1961) (received April 17, 1961)

Pion-Nucleon Interactions in the Region of the Higher Resonances

"In our opinion the minimum requirement for extrapolation is that the cross section in the physical region from which the extrapolation is made have the qualitative features of OPE (one-pion exchange). It is difficult to say whether the data of Anderson et al. (at $890 \mathrm{Mev} \pi$ - energies) satisfy 
this requirement. While the $\pi-\pi$ cross section may well behave as they indicate, the procedure from which this result is derived is open to serious objections."

220.S.Glashow and. M. Gell-Mann

Cal. Tech. Report CTSL-28, April 24, 1961

Gauge Theory of Vector Particles

221.S. Bergia and A. Stanghellini

Nuovo cimento 21, 155 (1961) (received May 10, 1961)

The Resonant Pion-Pion Model for the Nucleon Structure

"A theoretical model based on strong. $\pi-\pi$ interaction is compared with experimental data on nucleon form factors, and the determination of the parameters is discussed. "'.

222.K. Tanaka

Nuovo cimento 21, 169 (1961) (received.May 12, 1961)

Neutral Scalar $\bar{\sigma}$ Meson and the Mass Difference Between Muon and

\section{Electron}

223. J. Uretsky

Phys。Rev。123, 1459 (1961) (received May. 18, 1961)

$\underline{S-W a v e ~ P i o n-N u c l e o n ~ S c a t t e r i n g}$

224. C. Lovelace

Nuovo cimento 21, 305 (1961) (received May 18, 1961)

On the Existence of Solutions of the Pion-Pion Dispersion Equations. I。

"It is proved that the Chew-Mandelstam equations for pion-pion scattering with both $s$ and $p$ waves possess no exact solutions."

225. V. De Alfaro and B. Vitale

Phys. Rev。 Letters 7, 72 (1961) (received May 23, 1961)

Possibility of a 4-Pion Structure in the $\mathrm{T}=\mathrm{J}=1$ Pion-Pion

Scattering Amplitude

"... Let us analyze briefly how the many-pion configurations can be influenced by a resonance in the antisymmetric (both in isobaric spin and in configuration space) $\mathrm{T}=\mathrm{J}=1$ state. $\mathrm{n}=3$ : The maximum number of $\mathrm{T}=\mathrm{J} .=1$ pion gonds ("bi-pions") is represented in the configuration (11.1) represented by . This configuration contains three bonds, and has $\mathrm{T}=0$ and $\mathrm{J}=1$; it coincides with the enhanced 3 -pion state discussed by Chew (called "three-pion")。 $n=4$ : The maximum number of "bi-pions" is present in the configuration (2/1), represented by . It contains three bonds, has $T=1$ and $J$ odd. It can be described as a "three-pion" plus a spare pion, which leads to an immediate understanding of its $T=1$. In its lowest angular momentum state it has the same quantum numbers as the $\mathrm{T}=\mathrm{J}=1$ "bi-pion"'!!" 
226. B。 H。 Bransden and J。W. Moffat

Nuovo cimento 21, $505^{\circ}$ (1961) (received May 29, 1961)

Solutions of the Couplcd $s$ - and p-Wave Equations for Pion-Pion

Scattering

"A method is presented for solving numerically the coupled s- and p-wave equations for pion-piun scattering..:"?.

227. V. De Alfaro and B. Vitale

Nuovo cimento 21, 197 (1961) (received June 17, 1961)

Pion-Pion Interaction and Proton-Antiproton Annihilation at Rest

228. A。 P. Contogouris

Nuovo cimento 21,674 (1961) (received June 19, 1961)

Exchange Contribution: in Proton Compton Scattering

"...The effect of the two-pion intermediate states might be approxi-"

mated by simply adding to the Compton scattering amplitude a contribution in the form of a lowest-order perlurbalion term with exchange of "a partisle" of spin 0 , isospin $\mathrm{T}=0$, and mass delermined from the position of the pole."

229.A. Vo Efremov, M. Y. Tzu, and D. V. Shirkov

Dubna Report D-697, A 533 (submitted to Zhur. Eksptl。i Teoret, Fiz. 1961)

The Neutral Model for the Investigation of the Pion-Pion Scattering

230. F。 Zachariasen

Phys。Rev。Letters 7, 112, (1961) (received Jan。28, 1961)

Self-Consistent Calculation of the Mass and Width of the $\mathrm{J}=1, T=1$, $\pi-\pi$ Resonance

See also Eriatum in Phys. Rev。 Letters 7, 268 (1961): $\mathrm{M}_{\rho}=350 \mathrm{Mev}$ rather than $950 \mathrm{Mev}$.

231.T.Kotani, No Mishima, and M. Monda

Progr. Theoret. Phys。 (Kyoto) (to be published) (preprint received

by compiler June 1961)

Observation of the $\pi-\pi$ Resonance in the Pion Production Process

232.Y。Fujii

Nuovo cimento 21,684 (1961) (received July 5, 1961)

Some Consequences of Nuclear Forces Due to Pion-Pion Interaction

233. S. Minami

(Preprint received by compiler Aug. 31, 1961)

Three-Pion Resonance and High-Energy Phenomena

234. S. A: Bludman and J.A. Young

Lawrence Radiation Laboratory Report UCRL-9837, Aug. 23, 1961

Electromagnetic Production of Charged Vector Mesons 
"The Coulomb scattering, Compton scattering, bremstrahlung, and pair production of charged particles of spin one is investigated, in comparison with spin zero and one-half."

\section{R. Blankenbecler}

Phys. Rev。 (to be published) (preprint received by compiler Sept. 1961)

The Effect of the $3 \pi$ Resonance on the $2 \pi$ Resonance

"It is shown that the $4 \pi$ intermediate state in $\pi-\pi$ scattering plays an important pole in determining the position and perhaps even the existence of the $\pi-\pi$ resonance."

236. R. Blankenbecler and.J. Tarski

(Preprint received by compiler Sept, 1961)

Iso-Scalar Nucleon Structure

"The three-pion contribution to the iso-scalar nucleon structure is examined in dispersion.theory... We picture the process as being dominated by a low-energy $\pi-\pi$ resonance. The effects of a three-pion interaction are also included. If the $\pi-\pi$ resonance is at $10 \mu 2$ then a reasonable radius can be easily obtained. On the other hand if the resonance is above $20 \mu^{2}$ then a strong intrinsic three-pion resonance or bound state seems to be needed for agreement with experiment."

237. V. I. Ogievetski and I. V. Polubarinov Zhur. Eksptl。i Teoret。Fiz。41, 247 (1961)

Gauge-Invariant Formulation of the Neutral Vector Field Theory

\section{L. Schiff}

Phys. Rev. Jan. 15; 1962 (received Sept. 1961)

Particle-Theory Approach to Two-Pion and Three-Pion Systems

"The relation between the two-pion and three-pion resonances is discussed in terms of a model in which the motion of the pions is described by a partially relativistic Schrodinger-type wave equation, and the interaction between them is represented by a static potential. An attractive square-well potential that is almost strung enough to bind the di-pion gives a satisfactory account of the observed p-wave pion-pion scattering. If this potential is assumed to be

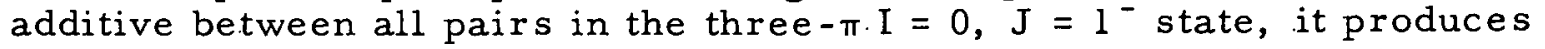
far too much binding to agree with observations. As an alternative to additivity, the interaction may be assumed to saturate, so that the total potential never exceeds that between any pair. of pions. It is found that the model provides a qualitative consistent explanation both of pion-pion scattering and the $3-\pi$. system if the pair interactions saturate or nearly saturate."

239. M. Gell-Mann

(Submitted to Phys. Rev。Sept. 13, 1961)

Symmetries of Baryons and Mesons

240. P. Carruthers

(To be submitted for publication)

Integral Equations for the Process $3 \pi \rightarrow 3 \pi^{*}$ 
"... Perhaps the most'suggestive remark made to date is: that of Chew, who pointed out that the antisymmetry of the isospin zero three-pion wave function under the interchange of any pair permits each pair to interact with the others through the strong $p$-wave isospin $1 \pi-\pi$ force... All graphs of this type are summed by a simple integral equation for the scattering amplitude $3 \pi \rightarrow 3 \pi^{*}$.

241.J.. J. Sakurai

Phys.. Rev. Letters 7, 355 (1961) (received Oct. 12, 1961)

Existence of Two $T \equiv 0$ Vector Mesons 


\section{Part II: LISTING BY TOPICS}

\section{A. General Theoretical Papers}

49. I. I. Pomeranchuk, Doklady Akad. Nauk S. S. S. R. 78, 889 (1951).

54. H. Miyazawa, Phys. Rev。 97, 1399 (1955).

59. S. Z. Belenkii and L. D. Landau, Uspekhi Fiz. Nauk 56, 309. (1955)

61. S. Z. Belenkii, Nuclear Phys. 2, 259 (1956).

63. I., Dyatlov, V. Sudakov, and K. Ter-Martirosyan, Soviet Phys. JETP $\underline{5}, 631$ (1957).

66. S. Z. Belenkii, Soviet Phys. JETP $\underline{5}, 952$ (1957)。

67. A. N. Mitra and R. P. Saxena, Phys. Rev. 108, 1083 (1957).

79. Y. Fujii, Progr. Theoret. Phys. (Kyoto) 21, 232 (1959).

93. M. Sugawara and A. Kanazawa, Phys. Rev. 115, 1310 (1959).

100. V. I. Rus'kin and P. A. Usik, Soviet Phys. JETP 11, 669 (1960).

104. S. Okubo, Phys. Rev. 118, 357 (1960).

105. Y. Fujii and S. Furuichi, Progr. Theoret. Phys. (Kyoto) 23, 251 (1960).

106.W. Frazer and J. Fulco, Phys. Rev, 117, 1603 (1960).

112. M. Baker and F。 Zachariasen, Phys。 Rev。 118, 1659 (1960).

114.G. F. Chew, Phys. Rev。 Letters $\underline{4}, 142$ (1960)。

118. G. F. Chew and S. Mandelstam, Phys。 Rev。 119, 467 (1960).

119. G. Chew, S. Mandelstam, and H. P. Noyes, Phys. Rev。 119, 478 (1960).

122. A. A. Ansel' $\mathrm{m}$, Soviet Phys. JETP 11, 1356:(1960).

123. V. A. Kolkunov, L。B. Okun, A. P. Rudik, and V. V. Sudakov, Soviet Phys. JETP 12, 242 (1961)。

137. B. W. Lee and M. T. Vaughn, Phys。Rev. Letters 4, 578 (1960).

147. Y. Miyamoto,..Progr。Theoret. Phys。(Kyoto) 24, 840 (1960).

148. A。 N. Mitra, R。 P. Saxena, and P. Narayanaswamy, Nuclear Phys. 20, 491 (1960).

152. Ding-Chang Hsien, Tso-hsiu Ho, and W. Zöllner, Soviet Phys..JETP $12,1165^{\circ}(1960)$.

156. Hung-yuan Chou, Soviet Phys. JEPT 13, 156.(1961).

158. Y. Simonov and K. A. Ter-Martirosyan, Soviet Phys. JETP 12,1003 (1961).

166. G. F. Chew, 1960 Rochester Conference, p. 273

168. J.W. Moffat, 1960 Rochester Conference, p. 355 
172. J.W. Moffat, Phys. Rev. 121, 926 (1961).

176. J. Bowcock, W. N. Cottingham, and D. Lurie, Phys. Rev. Letters 5, $386(1960)$.

177. H. Wong, Phys. Rev。 121, 289 (1960)。

178: Tso-hsiu Ho and Kuang-chao Chou, Soviet Phys. JETP 12, 1032 (1961).

181. G. F. Chew and S. Mandelstam, Nuovo cimento 19, 752 (1961).

186. Y. Fujii, Progr. Theoret. Phys. (Kyoto) 25, 441 (1961).

204. B. W. Lee and K. S. Cho, Nuovo cimento 20, 553 (1961)。:

211. M. Gell-Mann, Calif. Inst。 of Tech. Synchrotron-Lab. Report CTSL-20, March 1961:

215. B. H. Bransden and J. W. Moffat, Phys. Rev. Letters 6, 708 (1961).

216. G. F, Chew and S. C. Frautschi, Phys. Rev. 123, 1478 (1961).

217. B. Desai, Phys. Rev. Letters 6, 497 (1961).

224. C. Lovelace, Nuovo cimento 21, 305 (1961).

225.V. De Alfaro and B. Vitale, Phys. Rev. Letters 7, 72.(1961).

226. B. H. Bransden and J。W. Moffat, Naovo cimento 21, 505 (1961).

233. S. Minami (preprint)

235. R. Blankenbecler, Phys。Rev。 (to be published)

238. L. Schiff, Phys. Rev. (to be published)

239. M. Gell-Mann (submitted to Phys。Rev.).

240. P. Carruthers (to be submitted for publication).

\section{B. Pion-Nucleon Interaction}

\section{a. Experimental Papers}

1. L。 M. Eisberg, W. B. Fowler, R. M。 Lee, W. D. Shephard, R. P. Shutt, A. M. Thorndyke, and W. L。 Whittemore, Phys. Rev. 97, 797 (1955).

2. R. Cool, O. Piccioni, and D. Clark, Phys, Rev, 103, 1082 (1956).

3. G. Maenchen, W. B. Fowler, W. M. Powell, and R. W. Wright, Phys, Rev. 108, 850 (1957).

4. W. DoWalker, Phys. Rev。 108, 872 (1957).

7. M. Blau, C. F. Carter, and A. Perlmutter, Nuovo cimento, 14, 704 (1959).

8. W. Perkins III, J. Caris, R. Kenney, and V. Perez-Mendez, Phys. Rev. 118, 1364 (1960). 
9. V. G. Zinov, A. D. Konin, S. M. Kornechenko, and B. Pontecorvo, Soviet Phys。JETP 11, 1233, (1960)。

10. I. Derado, Nuovo cimento 15, 853 (1960).

13. E. Pickup, F。 Ayer, and E。O. Salant, Phys。Rev。 Letters 5, 161 (1960).

19. J. G. Rushbrooke and D. Radojcic, Phys. Rev. Letters $\underline{5}, 567$ (1960).

20. J.A. Anderson, V. Bang. P. Burke; D. Carmony, and N. Schmitz, Revs. Modern Phys. 33, 431 (1960).

23. E. O. Salant, E。 Pickup, D. K. Robinson, and B. A. Munir, Revs. Modern Phys。33, 534 (1961).

24. B. A. Munir, E. Pickup, D. K. Robinson; and E. O. Salant, Phys. Rev. Letters 6, 192.(1961)。

29. J.Anderson, V. Bang, P. Burke, D. Carmony, and N. Schmitz, Phys. Rev。 Letters 6, 365 (1961)。

30. Y。A. Batusov, S。A. Bunyatov, V. M. Sidorov, and V. A。 Yarba, Soviet Phys。JETP 40, 1528 (1961).

32. D. Robinson, B. Munir,: E. Pickup, and E. Salant, Bull。 Am。 Phys. Soc: 6, $301(1961)$.

33. A。 R. Erwin, R。 March。W. D. Walker, and E。 West, Phys。 Rev。 Letters $\underline{6}, 628$ (1961)。

34. W. Walker, H. Fechter, R. March, D. Lyon, P. Satterblom and A. Erwin, Bull. Am. Phys. Soc. 6, 311 (1961).

35. Do. Stonehill, C. Baltay, H。 Courant, W. Feckinger, E. C. Fowler, H. Kraybill, J. Sandweiss, J. Sanford, and J。Taft, Phys。Revo Letters. 6, 624 (1961)。

42. E. Pickup, D. K. Robinson, and E。O.Salant, Phys。Rev。 Letters 7, $192(1961)$.

43. A. Pévsner, K。 Kraerner, M. Nussbaum, P. Schlein, M。 Block, A. Kovacs, and C. Meltzer (preprint).

47. R. Barloutaud, C. Choquet, C. Gensollen, J. Heughebaert, A. Leveque, J. Meyer, and G. Viale; Conf. on Elementary Particles, Aix-en-Provence, France, Sept. 14, 1961.

- 48. J. Kirz, J.Schwartz, and R。Tripp, UCRL-9934, Nov。6, 1961, (submitted to Phys. Rev。) 


\section{b. Theoretical Papers}

50. O. Piccioni, 1952 Rochester Conference.

51. J. S. Kovacs, Phys. Rev. 93, 252 (1954).

52. A. N. Mitra and F. J. Dyson, Phys. Rev. 90, 372 (1953).

53. Marc Ross, Phys. Rev. 95, 1687 (1954).

56. F. J. Dyson, Phys。Rev。 99, $1037(1955) \%$

57. D. Ito and S. Minami, Progr. Theoret. Phys. (Kyoto) 14, 482 (1955).

58. G. Takeda, Phys. Revo 100, 440 (1955).

72. S. N. Gupta, Phys, Rev, 111, 1698.(1958).

74. V。 I。 Rus'kin, Soviet' Phys. JETP 9, 113 (1959).

77. C. Goebel; Phys. Rev. Letters 1, 337 (1958).

78. L。 B. Okun and I. Y. Pomeranchuk, Soviet Phys. JETP. 9, 207 (1959).

80. G. F. Chew and F。 E. Low, Phys。 Revo, 113, 1640 (1959).

82. K. Ishida, Progr. Theoret. Phys. (Kyoto) 25, 797 (1961).

85. A. A. Ansel'm and V. N。Gribov, Sov. Phys.JETP 10, 354 (1960).

87. V. I. Rus'kin, Soviet Phys. JETP 10, 74 (1960).

90. D. Ito, M. Yamazaki, T. Kobayashi, and K. Mori, Progr. Theoret. Phys. (Kyoto) 22, 448 (1959):

94. V. S. Barashenkov, Soviet Phys. JETP 10, 1038 (1959).

95. I. Sata, A. Takahashi, and Y. Ueda, Progr. Theoret. Phys. (Kyoto) 22, $617(1959)$.

96. A. D. Galanin, Soviet Phys. JETP 11, 177(1960).

98. V. S. Barashenkov, Nuclear Phys. 15, 486 (1960)。

108. J. Hamilton and W. So Woolcock, Phys, Rev。 $118,291(1960)$.

110. Bonsignori and Selleri, Nuovo cimento 15, 465 (1960).

113. T. Sakuma, Progr. Theoret. Phys. (Kyoto) 23, 810 (1960).

116.K. Kishida, A. Takahashi, and T. Jeda, Progr. Theoret, Phys. (Kyoto) 23, 731 (1961).

120. I。 M. Dremin, Soviet Phys. JE⿰氵 12, 94 (1961).

121. M. Cini and S. Fubini, Ann. Phys. 10, 352 (1960).

125. J. Bowcock, N. Cottingham, and D. Lurie, Nuovo cimento 16, 918 (1960)。

130. F. Selleri, Nuovo cimento 16, 775 (1960).

131.K. Itabashi, M. Kato, K. Nakagawa, and G. Takeda, Progr. Theoret. Phys: (Kyoto) 24, 529 (1960)。 
132. W. Frazer and J. Fulco, Phys。Rev. 119, 1420 (1960).

134. P. Carruthers and H. A。 Bethe, Phys. Rev。 Letters 4, 536 (1960).

139. M. Sugawara and A. Kanazawa, Phys. Rev。119, 2074. (1960).

142.S. C. Frautschi and J. D. Walecka, Phys. Rev。 120, 1486 (1960).

143. F. Salzman and G. Salzman, Phys. Rev. 120, 599 (1960).

144. Y. Batusov, S. Bunyatov, V. Sedorov, and V. Yarba, Soviet Phys. JETP 12, 354 (1961)。

146. V. S. Barashenkov, Nuclear Phys。22, 71 (1961).

153. A. V. Efremov, A. A. Meshcheryakov, D. V. Shirkov, and Hun-yuan Tzu, Nuclear Phys, 22, 202 (1961).

155. J. Hamilton and T. D. Spearman, Ann。 Phys. 12, 172 (1961).

159.S. C. Frautschi, Phys。Rev. Letters 5, 159 (1960).

160.S. Ishida, Progr. Theoret.Phys. (Kyoto) 24, 1262 (1960).

164. S. Sawada, Progr. Theoret. Phys。(Kyoto) 25, 83 (1960).

167. R. J.Eden, 1960 Rochester Conference, p. 219.

171.G.Salzman and F。 Salzman, 1960 Rochester Conference, p. 348.

174. J. Bowcock, N. Cottingham, and D. Lurie, Nuovo cimento 19 , 142 (1961).

175. D: . I。 Blokhintsev and Wang Yung, Nuclear Phys. 22, 410 (1961).

180. V.N. Strel'tsov, Soviet Phys。JETP 13, 802 (1961).

185.K. Ishida, Progr。Theoret. Phys. (Kyoto) 25, 294 (1960).

189. M. Yamazaki, Progr. Theoret Phỳs. (Kyoto) 25, 722 (1961).

190. P. Carruthers. Ann. Phys。14, 229 (1961).

193. P. Carruthers, Phys. Rev。122, 1949 (1961).

194. S. Sawada, $T_{0}$ Ueda, and M。 Yonezawa, Progr。Theoret. Phys. (Kyoto) 25, 873 (1961).

196. J. Hamilton, P. Menotti, T. D. Spearman, and W. S. Woolcock, Nuovo cimento 20, 519 (1961).

212. C. Goebel and H. J. Schnitzer, Phys, Rev。 123, 1021 (1961).

219. P. Carruthers, Phys。Rev. Letters 6, 567 (1961).

223.J.Uretsky, Phys。Rev。123, 1459 (1961).

231. T. Kotani, N。 Mishima, and M。 Monda, Progr。Theoret. Phys. (Kyoto) (to be published): 
C. Nucleon-Antinucleon Interaction

a. Experimental Papers

6. G. Goldhaber, W. B. Fowler, S. Goldhaber, T: F. Hoang, $\mathrm{T}$. Kalogeropoulous, and $\mathrm{W}_{\circ} \mathrm{M}$. Powell, Phys. Rev。 Letters 3, 181 (1959).

11. G. Goldhaber, S. Goldhaber, W. Lee, and A. Pais, Phys. Rev。 120, $300 .(1960)$.

15. J. Button, P. Eberhard, G。 R. Kalbfleisch, J. Lannutti, S. Limentani, and $G_{v}$ Lynch., and $B$. Maglić, $M_{0}$ L。 Stevenson, and NguyenXuong, 1960 Rochester Conference, $p_{0}: 166$.

16. S. Goldhaber, Go Goldhaber, W. Powell, and R. Silberberg, Phys. Rev。 121, 1525 (1961)。

22. G. Goldhaber and Wo Lee, Revs. Modern Phys. 33, 402 (1961).

39. B。C。 Maglić, G, R。 Kalbfleisch, and M. L。 Stevenson, Phys. Rev。 Letters 7, 137 (1961).

41. B. Maglik, L。 W. Alvarez, A. H。 Rosenfeld, and M。. L。 Stevenson, Phys。Rev。 Letters 7; 178 (1961)。

44. M. L。 Stevenson, L。W.Alvarez, 'B。C. Maglič, and A. H: Rosenfeld, Phys。Rev。(Jan。15, 1962).

45. N. Xuong and G。 R. Lynch, Phys。 Rev: Letters 7, 327 (1961).

\section{b. Theoretical Papers}

64. G. Sudarshan, Phys。 Rev, 103, 77.7 (1956).

69. T. Goto, Nuovo çimento 8, 625 (1958).

71. E. Eberle, Nuovo cimento 8, 610 (1958).

99. F. Cerulus, Nuovo cimento 14, 827 (1959).

109. V. M. Maksimenko, Soviet Phys。JETP 11, 469 (1960).

138. V。I. Gol'danskii and V. Mo Maksimenko,. Soviet Phys. JETP 12 , 584 (1961).

170.G。 Pinski, E。C。G。Sudarshan, and.K.T. Manhantappa, 1960 Rochester Conf., p. 173 .

192. G. Pinski, Physs Revo. Letters 6, 136 (1961).

203.J.G. Belifante, Phys。Rev. 123, 306 (1961).

227. V. De Alfaro and B. Vitale, Nuovo cimento 21, 197 (1961). 
D. Electromagnetic Structure.of.the Nucleon

\section{a. Experimental Papers}

26. D. N. Ol son, H. F。 Schopper, and R. R。 Wilson, Phys. Rev. Letters 6, $286(1961)$.

27. R. Hofstadter and R. Herman, Phys. Rev。 Letterș 6, 293 (1961)。

28. R. Hofstadter, C. Devries, and R. Herman, Phys. Rev. Letters 6, $290(1961)$.

37. R. M. Littauer, H。 F. Schopper, and R. R. Wilson, Phys. Rev. Letters 7. $141 .(1961)$.

38. R. M. Littauer, H. F。 Schopper, and R. R. Wilson, Phys. Rev。 Letters 7, $144(1961)$.

\section{b. Theoretical Papers}

68. Y. Nambu, Phys。Rev。106, 1366 (1957)。

70. G. F. Chew, R. Karplus, S. Gasiorowicz, and F. Zachariasen, Phys. Rev。110, 265 (1958)。

73. P. Federbush, M. L。 Goldhaber, and S. B. Treiman, Phys. Rev. $112,642(1958)$.

75. R.W. Huff, Phys. Rev。 112, 1021 (1958).

76. S. D. Drell, 1958 CERN Conf。, pp。20-33.

81. S. N. Gupta, Phys, Rev。111, 1436 (1958) and Phys. Rev. Letters. 2 , 124 (1959).

84. B. Bosco and V。 De Alfaro, Phys。Rev。115, 215 (1959).

86. W. R. Frazer and J。R. Fulco, Phys. Rev。 Letters 2, 365 (1959).

107. W. R。 Frazer and.J。R.Fulco, Phys。Rev。117, 1609 (1960).

129.E。V. Teodorovich, Soviet Phys. JETP 12, 334 (1961).

136. K. Hiida and N. Nakamishi, Progr. Theoret. Phys. (Kyoto) 24, 414,(1960).

184. J. Ball and D. Wong, Phys. Rev. Letters 6, 29 (1961).

207.S. Bergia, A. Stanghellini, S. Fubini, and C。Villi, Phys。Rev. Letters 6, 367 (1961).

221.S. Bergia and A. Stanghellini, Nuovo cimento 21, 155 (1961).

236. R. Blankenbecler and J. Tarski (preprint)。 


$$
\text { E. } K^{ \pm}-\text {Meson Decay }
$$

\section{a. Experimental Paper}

40. M, Ferro-Luzzi, D. H. Miller, J. J. Murray, A. H. Rosenfeld, and R. D. Tripp, Nuovo cimento (to be published)

\section{b. Theoretical Papers}

88. B. S. Thomas and W. G. Holladay, Phys, Rev。 11b, $13 \angle \dot{y}(1958)$.

92: S. Sawada and M。 Yonezawa, Progr. Theoret. Phys. (Kyoto) 22, 610 . (1959).

117. M. L. Good and W. G. Holladay, Phys. Rev。 Letters 4, 138 (1960).

126. N. N. Khuri and S. B. Treiman, Phys. Rev。 119, 1115 (1960).

133.R. F. Sawyer and K。C.Wali, Phys。Rev。.119, 1429 (1960).

140.A. N. Mitra, Nuclear Phys。 18, 502 (1960).

179.E.Loman, S。 Morris, E。 Irwin, Jr., and T. Truong, Ann. Phys。13, 359 (1961).

200.R。G. Sachs and B. Sakita, Phys. Rev。 Letters 6, 306 (1961).

208.K. Kawarabayashi, Nuovo cimento 20, 1030 (1961).

210.S.Sawada, T. Ueda, and M. Yonezawa, Progr。Theoret. Phys. (Kyoto) 25, 868 (1961)。

213.P. Budini and L. Fonda, Phys。Rev. Letters 6, 419 (1961).

214.J.Wolf and W. Zöllner, Dubna Report D-703, 1961. 
F. Photoproduction

a. Experimental Papers

5. Alberigi, Bernardini, Querzoli, Salvini, Silverman, and Stoppini, 9th Annual Intern. Conf. on High Energy Phys., Kiev, Vol.' 1, $42(1960)$.

12. R. Gomez, H. Burkhardt, $M$. Daybell, $H_{0}$.Ruderman, M. Sands, and R. Talman, Phys. Rey. Lettẹs 5, 170 (1960).

25. K. Berkleman, G。 Cortellessa, and A. Reale, Phys. Rev. Letters $\underline{6}$, $234(1961)$.

b. Theoretical Papers

83. A. A. Ansellm and V. N. Gribov, Soviet Phys. JETP 9, 1345 (1959).

151. J. S. Ball, Phys. Revo Letters 5, 73 (1960).

161.J. D. Soloviev, Soviet Phys. JETP 13, 418 (1961).

162. B. De Tollis, E. Ferrari, and.H. Muczek, Nuovo cimento 18, 198 (1960).

163. M. Gourdin, D. Lurie, and A. Martin, Nuovo cimento 18, 933 (1960).

173. I.، D. Solov'ev, G. Bialkowski, and A。 Jurewicz, Soviet Phys. JETP (1961).

187. M. Kato, Progr. Theoret. Phys. 25, 493 (1961).

188. J. Uretsky and T. Palfrey, Jr. Phys. Rev。 121, 1798 (1961).

191. N. F. Nelipa; Soviet Phys. JETP 13, 766 (1961).

195. M. Kawaguchi, M. Miyamoto, and Y. Fujii, Nuovo cimento 20, 408 (1961).

202. M. Bassetti, Nuovo cimento 20, 803 (1961).

206. B. DeTollis and A. Verganelakis, Phys. Rev. Letters 6, 371.(1961).

228. A. P. Contogouris, Nuovo cimento 21, 674 (1961).

229. A. V. Efremov, M。 Y. Tzu, and D. V. Shirkov, Dubna Report D-697, (1961)。

234.S. A. Bludman and J。A. Young, UC.RL-9837, Aug. 23, 1961. 


\section{G. Heavy Mesons}

\section{a. Experimental Paper}

17. N. E. Booth, O. Chamberlain, and E。 H。 Rogers, Nuovo cimento 19, 853, (1961)。

\section{b. Theoretical Papers}

55. M. $\mathrm{H}_{0}$ Johnson and $\mathrm{E}_{\circ}$ Tellcr, Phys。 Rev。 98, 783 (1955)。

60. H. Duerr and E. Teller, Phys。Rev. 101, 495 (1956).

62. E。 Teller, Proc。1956 Rochester Conf., p. VII-18.

65. $\mathrm{H}_{a}$ Duerr, Phys。 Rev。 103, 469. (1956)。

91. Y. Yamaguchi', Progr。Theoret, Phys。(Kyoto) Suppl. 11, (1954).

97. M. Ikeda, S. Ogawa, and Y. Ohnuki, Progr。 Theoret. Phys. (Kyoto) 22, 715 (1959).

$101 . J_{0}$ 'Wess, Nuovo cimento 15, 52 (1960).

102.K.Igi, Progr。Theoret, Phys. (Kyoto) 23, 170 (1960).

124。J。Sakurai, Ann。 Phys。11, 1 (1960).

145.S. Kamefuchi, Nuclear Hhys, 18, 641 (1960).

165. A. Komar and A. Salam, Nuclear Phys. 21, 624 (1960)。

169.Y.Ohnuki, 1960 Rochester:Conf。, p. 843.

199. Y. Neeman, Nuclear Phys。26, 222 (1961).

205.I.Y.Kobzarev, L。B。Okun, and I。 Y。Pomeranchuk, Zhtar。 Eksptl. i Teoret. Fiz. 41, 495 (1961).

209. A. Salam and J, C, Ward, Nuovo cimento 20, 419 (1961).

218. M. Gell-Mann and F。 Zachariasen, Phys. Rev。 124, 953 (1961).

220.S. Glashow and M. Gell-Mann, Cal-Tech. CTSL-28, Apr. 24, 1961:

222.K. Tanaka, Nuovo cimento $21,169 .(1961)$.

230. F. Zachariasen, Phys. Rev。 Letters 7.112 (1961).

237. V. I. Ogievetski and.I. V. Polubarinov, Zhur。Eksptl。i Teoret.Fiz。 $\underline{41}, 247$ (1961).

241.J.J.Sakurai, Phys。Rev。Letters 7, 355 (1961)。 
H. Nucleon-Nucleon Interaction

\section{Theoretical Papers}

103. R. Hagedorn, Nuóvo ciménto 15, 246 (1960).

111. M. L. Goldberger and R. Oehme, Ann. Phys. 10, 153 (1960).

127. D. Amati, E. Leader, and B. Vitale, Nuovo cimento 17, 68 (1960).

128.J. J。Sakurai, Nuovo cimento 16, 388 (1960).

135.J.J.Sakurai, Phys. Rev. 119, 1784 (1960).

141.G. Breit, Phys。Revo 120,287 (1960), and Proc,Natl.Acad. Sci, , U.S. 46, 746 (1960).

149. D. Amati, E. Leader, and B. Vitale, Nuovo cimento 18, 409 (1960):

150. P. A. Usik and V。I. Rus'kin, Soviet Phys。JETP 12, 1200 (1961).

154. D. Amati, E. Leader, and B. Vitale, Nuovo cimento 18, 458 (1960).

157. V. S. Barashenkov, Soviet. Phys. JETP 13, 925 (1961).

197. S. Ohnuma, Progr. Theoret. Phys. (Kyoto) 25, 847 (1961)。

232. Y. Fujii, Nuovo cimento 21, 684 (1961).

$$
\text { I. Proton-Deuteron Interaction }
$$

\section{a. Experimental Papers}

14. A. Abashian, N. Booth, and K. Crowe, Phys。Rev。'Letters 5, 258 (1960)。

21. N. E. Booth, A. Abashian, and K. M. Crowe, Revs. Modern Phys. 33, 393 (1961).

31. Y.K. Akimov, V。I.Komarov, K, S. Marish, O。 V. Savchenko, and L. M. Soroko, Soviet Phys. JETP 13, 1073 (1.961)..

36. N. E. Booth, A. Abashian, and K. Crowe, Phys. Revo Letters ?, 3 (1961)。

46. Yu K. Akimov, V。 I. Komarov, Ko. So. Marish, O. V. Savchenko and L. M. Soroko, Dubna Report D-714 1961。

\section{b. Theoretical Papers}

183. Tubis and Uretsky, Phys. Rev。 Letters 5, 513 (1961).

198.J.G. Taylor, Phys. Rev。 Letters 6, 237 (1961).

201.T.N. Truong, Phys, Rev, Letters 6, 308 (1961). 


\section{J.Kaon-Nucleon Interaction \\ Theoretical Paper}

182. B.W. Lee, Phys。 Rev. 121, 1550 (1960).

$$
\text { K. Lack of Evidence }
$$

\section{a. Experimental Paper}

18. T. F。 Hoang, Phys. Rev. 121, 1523 (1961).

b. Theoretical Papers

89. V. S. Barashenkov. and V. M. Maltsev, Soviet Phys. JETP 10, 630 (1960). 115. A. I. Lebedev and V. A. Petrun'kin, Soviet Phys. JETP 11,962 (1960). 


\section{Part III: ALPHABETICAL.LISTING:OF AUTHORS} with Reference numbers

Abáshian, A.

Akimov, Yu.K.

Alberigi

Alvarez, L. W.

Amati, D.

Anderson, J.A.

Ansel'm, A. A.

Ayer, F.

Baker, $M$.

Ball, J.o S.

Baltay, C.

Bang, V.

Barashenkov, V.S。

Barloutaud, R.

Bassetti, $M$ 。

Batusov, Y。

Belenkii, S. Z.

Belifante, J。G.

Bergia, S.

Berkleman, K.

Bernardini

Bethe, H。A.

Bialkowski, G.

Blankenbecler, $R$.

Blau, M.

Block, M.

Blokhintsev,

Bludman, S. A.

Bonisignori,

Booth, N.

Bosco, B.
$14,21,36$

31,46

5

$41, .44$

$127,149,154$

20, 29 .

$83,85,122$

13

112

151,184

35

20,29

$89,94,98,157$

47

202

30,144

$59,61,66$

203

207, 221

25

5

134

173

235,236

7

43

175

234

110

$14,17,21,36$

84 


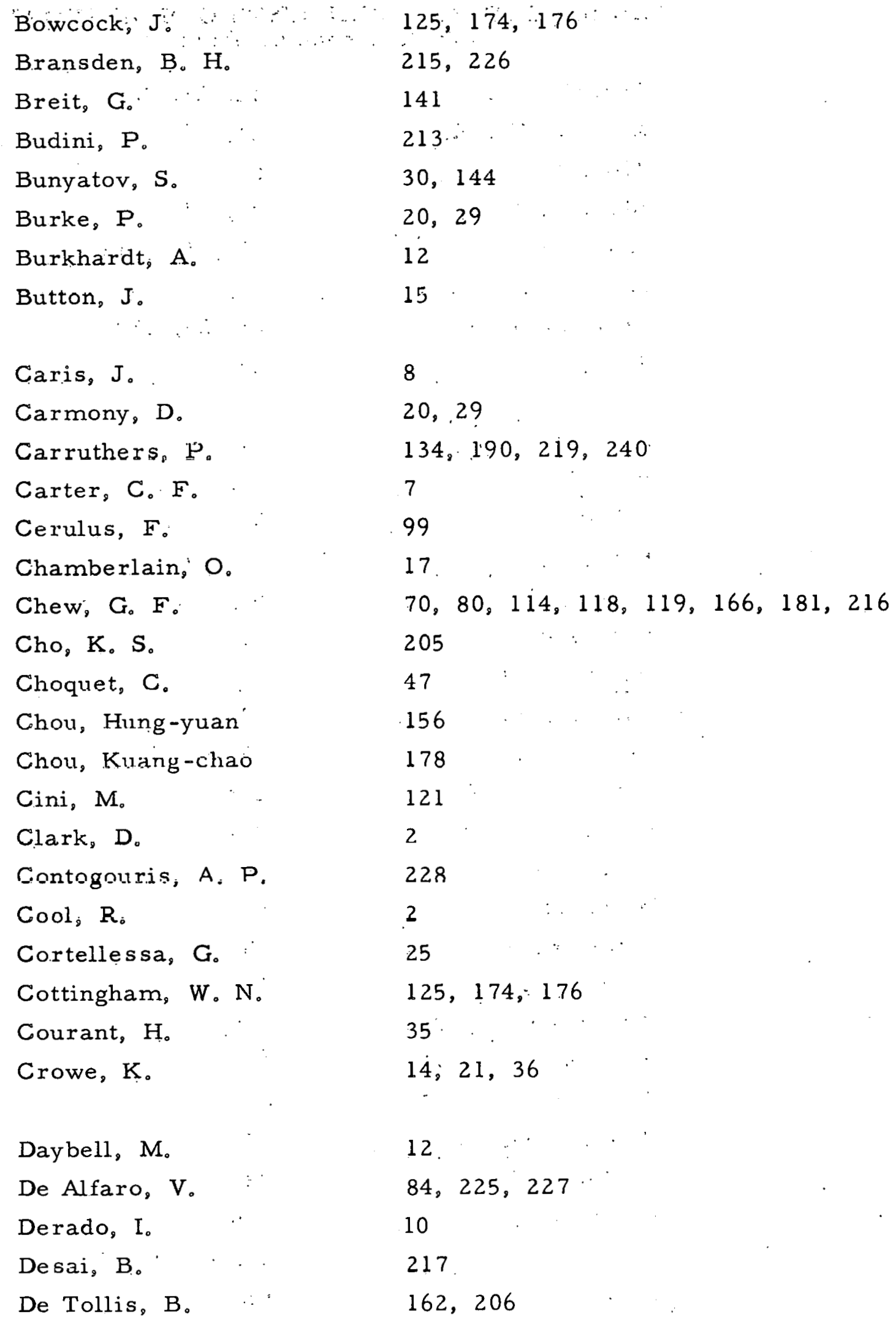


$\because \quad . \quad \cdots \quad-55-$

UCRL-9999

Devries, C.

Drell, S。 D。

28

Dremin, I. $M_{\leftarrow}$

Duerr, $\mathrm{H}_{\text {. }}$.

Dyatlov, I.

Dyson, F.J.

76

120

60,65

63.

52,56

Eberhard, P.

15

Eberle, E.

71

Efremov, A。 V.

153,229

Eden, R. J.

167

Eisberg. L。 $M$ 。

1

Erwin, A. R。

33,34 .

Fechter, $\mathrm{H}$ 。

34

Federbush, P.

73

Feqrari, E.

162

Ferro-Luzzi, M.

40

Fickinger, $W$ 。

35

Fonda, L.

213

Fowler, E.C. 35

Fowler, W。 B。

$1, .3,6$

Frautschi, S。C.

$142,159,216$

Frazer, W.

$86,106,107,132$

Fubini, S。

121,207

Fujii, Y。

$79,105,186,232$

Fulco, J。

$86,106,107,132$.

Euruichi, S。

105

Galanin, A. D.

96

Gasiorowicz, S.

70

Gell-Mann, M.

$211,218,220,238$

Gensollen, C.

47

Glashow, S.

220 
Goebel, C.

Gol'danskii, V. I.

Goldberger, M. L.

Goldhaber, G.

Goldhaber, $\mathbf{S}$.

Gomez, R.

Good, M: L.

Goto, $T$.

Gourdin, $\mathbf{M}$.

Gribov, V. N.

Gupta, S. N.

Hagedorn, $\mathbf{R}$.

Hamilton, J .

Herman, R.

Heughebaert, $J$.

Hiida, $K$.

Ho, 'l'so-hsiu

IIuaing, T. $\Gamma$.

Iofstadter, R.

Holladay, W. G.

Hsien, Ding-chang

Huff, R." $W$.

Igi, $\mathrm{K}$.

Ikeda, $\mathrm{M}_{\text {。 }}$

Irwin, $\mathrm{E}$, $\mathrm{Jr}$ 。

Ishida, K.

Ishida, $\mathbf{S}$.

Itabashi, $\mathrm{K}$.

Ito, D.

Jurewicz, A.

Johnson, M. H.
77,212

138

73,111

6, 11, 16, 22

$6,11,16$

12

117

69

163

83,85

72,81

103.

$108,155,196$

27,28

47

136

152,178

6,18

27, 28

88,117

152

75

102

97

179

$82,116,185$

160

131

57,90

173

55 
Kalbfleisch, G. R.

Kalogeropoulous, $T$.

Kamefuchi, S.

Kanazawa, A.

Karplus, $R$.

Kato, $M$.

Kawaguchi, M.

Kawarabayashi, K.

Kenney, R.

Khuri, N. N.

Kirz, J。

Kobayashi, $T$ 。

Kobzarev, I. Y.

Kolkunov, V。A.

Komar, A.

Komarov, V.I.

Korenchenko, S. M:

Konin, A. D。

Kotani, $T$ 。

Kovacs, A.

Kovacs, J。S.

Kraemer, K.

Kraybill, $\mathrm{H}_{\text {。 }}$

Landau, L. D.

Lannutti, J .

Leader, $E$.

Lebedev, A. I.

Lee, $B$. W. .

Lea, R. M.

Lee, W。

Leveque,' $A$.

Limentani, S.

Littauer, R. M.
15,39

6

145

93,139

70

131,187

195

208

8

126

48

90

205

123

165

31,46

9

9

231

43

51

43

35

59

15

$127,149,154$

115 .

$137,182, .205$

1.

$11, .22$

47

15

37,38 
Loman, $E_{0}: \vdots$ : :

Lovelace, C。 $\therefore$

Low, F。E。 : :

Lurie, D。

Lynch, G.

Lyon, D.

Maenchen, G。

Maglić, B.

Malcoimenlco, V: M。

Maltsev, Vo $M_{0}$,

Mandlestam,S。

Manhantappa, $K_{0}, T_{\text {。 }}$

March, $R$ 。

Marish, K.S.

Martin, A.

Meltzer, C.

Menotti, P.

Meshcheryakov, V.

Meyer, $J$ 。

Miller. Do $H$ 。

Minami。S.

Mishima, N.

Mitra, A。 N。

Mori, K.

Morris, S。

Miyamoto, :M:

Miyazawa, $\mathrm{H}$ 。

Moffat, $J$ 。W

Monda, M。

Muczek, H.

Munir, B.A。

Murray, $J$ 。
17.9

224

80

$125,163,174 ; 176$

15, 45

34

3

$15,39,41,44$.

109,138

89

$118,119,18,1$

170

$33,: 34$

31,46

163 .

43

196

153

47

40

57,233

231

$52,67,140,148$

90

179

195

54

168, I 12, 215, 226

231

162

$23,24, .32$.

40 


\begin{tabular}{|c|c|}
\hline Nakagawa, K. & 131 \\
\hline Nakanishi, No & 136 \\
\hline Nambu, Y & 68 \\
\hline Narayanasiwamy, $P$. & 148 \\
\hline Neeman, $Y_{0}$ & 199 \\
\hline Nelipa, N. F。 & 191 \\
\hline Noyes, H. P。 & 119 \\
\hline Nussbaum, $M_{\text {. }}$ & 43 \\
\hline Ugawa, ș. & 97 \\
\hline Ogievetski, Vo E. & 237 \\
\hline Ohnuma, S. & 197 \\
\hline Ohnuki。 Y. & 97 \\
\hline Okubo, S. & 104 \\
\hline Okun, L。B。 & $78,123,205$ \\
\hline Olson, D. No & 26 \\
\hline Pais, A. & 11 \\
\hline Palfrey, $T_{\circ}$ & 188 \\
\hline Perez Mendez, V。 & 8 \\
\hline Perkins, W. III & 8 \\
\hline Perimutter, $A_{0}$ & 7 \\
\hline Petrun'kin, Vo $A_{0}$ & 115 \\
\hline Pevsner, $A$. & 43 \\
\hline Piccioni, O. & 2,50 \\
\hline Pickup, E。 & $13,23,24,32,42$ \\
\hline Pinski, $G_{0}$ & 170,192 \\
\hline Polubarinov, I. V. & 237 \\
\hline Pomeranchuk, I。 I. & $49,78,205$ \\
\hline Pontecoryo, B. & 9 \\
\hline Powell, W. M。 & $3,6,16$ \\
\hline
\end{tabular}




\begin{tabular}{|c|c|}
\hline Radojcic, D. & 19 \\
\hline Reale, A. & 25 \\
\hline Robinson, D. K. & $23,24,32,42$ \\
\hline Rogers, E. H. & 17 \\
\hline Rosenfeld, A. H. & $40,41,44$ \\
\hline Ross, Marc & 53 \\
\hline Ruderman, $H_{。}$ & 12 \\
\hline Rudik, A. $P_{0}$ & 123 \\
\hline Rushbrooke, J。G. & $19^{\circ}$ \\
\hline Rus'k.ing V。I. & $74,87,100,150$ \\
\hline Sachs, $R_{0} G_{0}$ & 200 \\
\hline Sakita, B. & 200 \\
\hline Sakuma, $\mathrm{T}$. & 113 \\
\hline Sakurai, $J_{\circ} J_{\circ}$ & $124,128,135,241$ \\
\hline Salam, A. & 165 \\
\hline Saiant, E.O. & $13,23,24,32,42$ \\
\hline Salvini & 5 \\
\hline Salıinàn, $\Gamma$ & 143,171 \\
\hline Salzman, $G_{0}$ & 143,171 \\
\hline Sands, $M_{c}$ & 12 \\
\hline Sandweiss, J. & 35 \\
\hline Sanford, J. & 35 \\
\hline Sato, I . & 95 \\
\hline Satterblom, $P$ & 34 \\
\hline Savchenko, O. V. & 31,46 \\
\hline Sawada, S. & $92,164,194,210$ \\
\hline Sawyer, R. F。 & 133 \\
\hline Saxena, $R . P$. & 67,148 \\
\hline Schiff $L_{\circ}$ & 238 \\
\hline Schlein, $P_{\text {。 }}$ & 43 \\
\hline Schmitz, N。 & 20,29 \\
\hline
\end{tabular}


Schnitzer, $H$. J。

Schopper, H. F.

Schwartz, J. .

Sidorov, V.

Selleri, F.

Shephard, W。D.

Shirkov, D. V.

Shutt, R。 P.

Silverberg, $R$.

Silverman

Simonov, $Y$.

Soloviev, J. D.

Soroko, L. M.

Spearman, T. D.

Stanghellini, A.

Stevenson, M. L.

Stonehill, D。

Stoppini,

Strel'tsov, V. N.

Sudakov, V。

Sudarshan, G.

Sugawara, $M$.

Taft, $H_{0}$

Takahashi, A.

Takeda, G.

Talman, R.

Tanaka, K.

Tarski, J.

Taylor, J。G.

Teller, E。

Tepdorovich, E. V.

Ter-Martirosyan, $K$.
212.

$26,37,38$

48 .

30,144

110,130

1

153

1

16

5.

158.

161,173

$31, .46$

155, 196

207,221

$15,39,41,44$

35

5

180

63,123

64,170

93,139

35

95,116

58,131

12

222

236

198

$55, .60,62$

129

63,158 
Thomas, B. S.

Thorndyke, A: $M$ 。

Treiman, S。B.

Tripp, R。 D:

Truong, $\mathrm{T}$ 。 $\mathrm{N}$.

Tubis

$\mathrm{Tzu}$, Hun-yuan

Ueda, $T$.

Uedl, $Y$.

Uretsky, J 。

Usik, $P$. A.

Vaughn, $M$ 。 $T$ 。

Verganelakis, A.

Viale, G.

Villi, C.

Vilale, $D_{0}$.

Walecka, J。D.

Wali, K。C.

Walker, W. D.

Wess $\mathrm{J}_{\text {。 }}$

Weot, E。

Whittemore, W。 L.

Wilson, $R$ 。 $R$ 。

Wolf, J。

Woolcock, W.S.

Wong, D.

Wong, $\mathrm{H}$.

Wright, R.W.

Xuong, N. H.
88

1

73,126

40,48

179,201

183

153

194,210

25,116

$183,188,223$

100,150

137

206

47

207

$127,119,151,225,227$

142

133

$4,33,34$

1.01

33

1

$26,37,38$

214

108,196

184

177

3

15,45 


\author{
Yamaguchi, Y。 \\ -Yamazakis M. \\ Yarba, V. \\ Yonezawa, $M$ 。 \\ Young, J。A. \\ Yung, Wang \\ Zachariasen, F. \\ Zinov, V. G. \\ Zöllne $r, W$.
}

91

90,189

144

$92,194,210$

234

175

$70,112,218,230$

9

152,214 
This report was prepared as an account of Government sponsored work. Neither the United States, nor the Commission, nor any person acting on behalf of the Commission:

A. Makes any warranty or representation, expressed or implied, with respect to the accuracy, completeness, or usefulness of the information contained in this report, or that the use of any information, apparatus, method, or process disclosed in this report may not infringe privately owned rights; or

B. Assumes any liabilities with respect to the use of, or for damages resulting from the use of any information, apparatus, method, or process disclosed in this report.

As used in the above, "person acting on behalf of the Commission" includes any employce or contractur of the Commission, or employee of such contractor, to the extent that such employee or contractor of the Commission, or employee of such contractor prepares, disseminates, or provides access to, any information pursuant to his employment or contract with the Commission, or his employment with such contractor. 


\title{
ADDENDUM \\ to the \\ BIBLIOGRAPHY ON PION-PION INTERACTION
}

M. Lynn.Stevenson

\author{
Lawrence Radiation Laboratory \\ University of California \\ Berkeley, California \\ July 1962
}

I wish to express my sincerest regrets to the authors of the papers contained in this addendum for overlooking their work. in the original bibliography. If there are still further omissions, please inform.me of them so that a further addendum can be compiled. No attempt has been made to update this bibliography beyond November 7, 1961.

The numbers on these references indicate the positions they should have had in the original chronological listing of the bibliography.

Experimental Papers

2.1 W. D. Walker, F。 Hushfar, and W。D. Shepard

Phys. Revo 104, 526 (1956) (received July.2, 1956)

$\pi^{-}$-p Interactions in the $1.0-$ Bev Region

4.1.W.A. Perkins, J。C.Caris, R。W.Kenney, E。A.Knapp, and

V. Perez-Mendez

Phys. Rev。Letters 3,56 (1959) (received June 1, 1959)

Pion Production by Pions

31.1 Yu. K. Akimov, O.V. Savchenko, and L。 M. Soroko

Soviet Physics JETP 14, 512 (1962) (received April 21, 1961)

Experimental Verification of the Charge-Independence Principle in the $\mathrm{d}+\mathrm{d} \rightarrow \mathrm{He}^{4}+\pi$ Reaction for $400-\mathrm{Mev}$ Deuterons

Theoretical Papers

57.1 W. Go. Holladay

Phys。Rev, 101, 1.198 (1955) (received Aug。 5, 1955)

Nucleon Anomalous Moments via Pion-Pion Attraction

57.2 W. Go Holladay

Phys。Rev。101, 1202 (1955) (received Aug。 5, 1955)

Nucleon.Structure and the $n-p$ Mass Difference

68.1 . V. No Gribov

Nuclear Phys。 5, 653 (1958) (received June 18, 1957)

Angular Distribution in Reactions Involving the Formation of Three

Low-Energy Particles, with. Application to $\tau^{+}$-Meson Decay


71.1.V. I. Goldanskii and Ya.A. Smorodinskii, Soviet Phys. JETP 9, 1387 (1959) (received Apr. 4, 1959) Singularities of the $\bar{S}$ Matrix and the $\rho^{0}$ Meson

90.1 L. So Rodberg, Phys. Rev。 Letters 3, 58 (1959) (received June 1, 1959) Pion Production and the Pion-Pion Interaction

122.1.L. M. Brown and F. Calogero, Phys. Rev。Letters 4, 315 (1960) (received Feb. 5, 1960) Effects of Pion-Pion Interactions in Electromagnetic Processes

138.1 L。 M. Brown and $F$. Calogero Phys。Rev, 120, 653 (1960) (received May 3, 1960) Pion-Pion Interaction in Electromagnetic Processes

148.1 D. J. Blohincev

Nuovo cimento 18, 193 (1960) (received July 18, 1960) $\pi \pi$ Interaction in Peripheral $\pi N$ Collisions

205.1 I. Y. Kobzarev and. L. B. Okun Soviet Phys. JETP 14, 358 (1962) (received February. 28, 1961)

213.1 V。 G。 Zinov, A。 D。 Konin, S. M. Korenchenko, and B. Pontecorvo Soviet Phys。JETP 9, 1386 (1959) (received March 23, 1959) Possible Method of Search for the $p^{0}$ Meson

214.1 A. A. Anselm, V. N. Gribov, G. S. Denilov, J。T. Dyatlov, and V. M. Shekhter

Soviet Phys。JETP 14, 444 (1962) (received March 24, 1961) On the Maximum Value of the Coupling Constant in. Field Theory

215.1 V。 J. Rus'kin and. D. S. Chernavskii Soviet Phys。JETP 14, 451 (1962) (received March 27, 1961) Application of the Pole Method to the Analysis of Experimental Data on $\pi p$ Interactions

$216.1 \therefore$ A. D. Golanin and A。 F. Graslin Soviet Phys。JETP 14, 454 (1962) (received March 30, 1961) Pion-Nucleon Ampitude with Account of $\pi-\pi$ Interaction

237.1 V. I. Ogievetskii and I. V. Poluberinov Soviet Phys。JETP 14, 179 (1962) (received February 16, 1961) Gauge-Invariant Formulation of Neutral Vector Field Theory 